\title{
Force-Free Magnetohydrodynamic Waves: Non-Linear Interactions and Effects of Strong Gravity
}

\author{
Parker Troischt \\ Department of Physics and Astronomy, University of North Carolina, Chapel Hill, NC 2759\$ \\ Christopher Thompson \\ CITA, 60 St. George St., Toronto, ON, M5S $3 H \notin$
}

\begin{abstract}
The propagation and non-linear interactions of magnetohydrodynamic waves are considered in the force-free limit, where the inertia of the conducting matter which enforces the MHD condition $\mathbf{E} \cdot \mathbf{B}=0$ can be neglected in comparison with the inertia of the electromagnetic field. By extending the analysis beyond the WKB approximation, we are able to study the non-linearities induced by a gravitational field. We treat the perturbed electromagnetic field as a fluid of infinite conductivity. We calculate the scattering of a torsional (Alfvén) wave by a gravitational potential, and demonstrate a nonlinear coupling with a compressive (fast) wave which is second order in the amplitude of the Alfvén wave. In a cylindrically symmetric spacetime with slow rotation, the coupling is second order in $g_{t \phi}$ and first order in the amplitude of the wave. We also give a fresh analysis of the non-linear interactions between compressive and torsional waves in Minkowski space, with a focus on the relative strengths of their three- and four-mode interactions. In contrast with non-relativistic magnetofluids, the effects of compression are always present. In the case of colliding fast waves, a net displacement of the field lines across (at least) one of the colliding wavepackets is shown to have a strong effect on the outgoing waveform, and to have a qualitatively different interpretation than was previously suggested for colliding Alfvén waves. Finally, we show how spacetime curvature modifies the collision between two torsional waves, in both the weak- and strong-field regimes.
\end{abstract}

PACS numbers: Valid PACS appear here

Submitted to PRD 11 May 2004; accepted 18 August 2004

\section{INTRODUCTION}

Magnetohydrodynamics can be studied in the ultra-relativistic limit, where the energy density in the conducting matter which enforces $\mathbf{E} \cdot \mathbf{B}=0$ is much less than in the electromagnetic field itself. The Euler equation reduces to the force-free equation $J^{\mu} F_{\mu \nu}=0$, which provides a useful starting point for investigating some aspects of the dynamics of accreting and outflowing matter around compact stars, and classical gamma-ray bursts (e.g. [1], 2], [3], [4]). In this regime a uniform magnetofluid supports two modes: incompressible torsional waves analogous to Alfvén waves in non-relativistic magnetohydrodynamics (MHD), and a compressible wave analogous to the fast mode. Both of these modes have exact non-linear solutions in a uniform magnetic field in Minkowski space, which means in turn that there is no spontaneous decay of one type of mode into the other.

This fundamental property of a relativistic magnetofluid changes in the presence of a strong gravitational field. We focus in this paper on the effect of spacetime curvature on the interactions between torsional and compressive MHD modes. There is a non-linear interaction if the background spacetime is static, which appears at linear order if it is slowly rotating. We analyze the effect first in the weak-field regime, and consider both spherically and cylindrically symmetric gravitational fields. As a particular example where strong-field effects are important, we also consider the case of a uniform magnetofluid immersed in the spacetime of a black string.

Our main goal here is to explore the fundamentals of the interactions between MHD waves and gravitational fields. Although the scattering of gravitational waves, scalar waves, and vaccum electromagnetic waves have long been studied in black hole spacetimes [5], analogous work on force-free magnetohydrodynamics has focused on the short-wavelength limit 2]. There are two well-established astrophysical contexts in which the dynamics may be well described, in a first approximation, by a relativistic force-free fluid: the magnetosphere of a bursting magnetar (e.g. [6]); and the polar regions of an accreting black hole [1]. We restrict ourselves, in this paper, to the simplest case where the background magnetofluid does not maintain a large-scale current (as it does surrounding a Kerr black hole).

*Electronic address: troischt@physics.unc.edu

†Electronic address: thompson@cita.utoronto.ca 
In a dynamic situation, a fluid formulation of force-free magnetohydrodynamics provides a physically transparent set of variables. Two such formalisms have been developed, by Achterberg [7] and Thompson \& Blaes []. in fact, dual versions of each other, and are connected by interchanging the dynamical role of the Lagrangian fluid coordinates and the Eulerian coordinates of the background spacetime. In reviewing these formalisms, we generalize the second to an arbitrary curvilinear coordinate system.

Another of our goals is to extend the discussion of non-linear mode interactions in force-free MHD, begun in [3]. We examine in more detail how obliquely propagating Alfvén modes and fast modes will perturb each other, including the effects of spacetime curvature. The effect of field line wandering on mode collisions has received considerable attention in recent years, in the case of non-relativistic and incompressible Alfvén turbulence. We examine its effect on the collisions between compressive (fast) modes in a relativistic magnetofluid in flat Minkowski space. In contrast with the case of colliding Alfvén waves, the effect of a net field-line displacement cannot be expressed in kinematic terms through a zero-frequency component of one of the colliding waves.

The sections are organized as follows. In Section II, we given an overview the electric and magnetic lagrangian fluid formulations of force-free MHD. As a toy example, we construct the solution for the uniform magnetic field in a spherically symmetric spacetime (with non-vanishing Ricci tensor). In Section III we review the normal modes of a uniform, force-free magnetofluid. Section IV is devoted to the scattering of a torsional wave in a curved spacetime: we first consider the propagation of such a wave in a general, cylindrically symmetric spacetime; and then consider the scattering of such a wave in a shallow, spherical gravitational potential. Section V generalizes the results of the previous section to the case of two colliding, axisymmetric torsional modes in a gravitational field. The final Section VI considers the more general case of collisions between non-axisymmetric MHD modes in Minkowski space, focusing on the three-mode couplings of two colliding Alfvén and fast waves.

\section{LAGRANGIAN FORMULATIONS OF ULTRARELATIVISTIC MHD}

The equations describing the behavior of ideal fluids coupled to electromagnetic fields in curved spacetimes are inherently very complicated. A significant simplification is obtained by assuming that the spacetime metric is fixed; i.e., that the mass-energy of the fluid can be neglected in comparison with that of the source of gravity. In this paper we make a second simplification: the role of the charged matter fields is restricted to a source of electric currents which cancel off the electric field in the fluid rest frame. The mass-energy of these matter fields is, in other words, negligible in comparison with that of the electromagnetic field.

We begin by summarizing the two Lagrangian fluid formulations of relativistic, force-free MHD.

\section{A. MHD equations in the force-free limit}

The covariant Maxwell Equations are

$$
4 \pi J^{\mu}=\nabla_{\nu} F^{\mu \nu}=\frac{1}{\sqrt{-g}} \partial_{\nu}\left(\sqrt{-g} g^{\mu \alpha} g^{\nu \beta} F_{\alpha \beta}\right)
$$

and

$$
\nabla_{\alpha} F_{\mu \nu}+\nabla_{\nu} F_{\alpha \mu}+\nabla_{\mu} F_{\nu \alpha}=\partial_{\alpha} F_{\mu \nu}+\partial_{\nu} F_{\alpha \mu}+\partial_{\mu} F_{\nu \alpha}=0
$$

When the only force acting on the charged matter in a local inertial frame is the Lorentz force, the dynamics of the electromagnetic field is described by the force-free equation

$$
J^{\mu} F_{\mu \nu}=\frac{1}{4 \pi} \nabla_{\alpha} F^{\mu \alpha} F_{\mu \nu}=0
$$

in combination with

$$
F_{\mu \nu} \widetilde{F}^{\mu \nu}=0
$$

Here

$$
\widetilde{F}^{\mu \nu}=\frac{1}{2 \sqrt{-g}} \varepsilon^{\mu \nu \alpha \beta} F_{\alpha \beta}
$$

is the dual of the electromagnetic field tensor, expressed in terms of the antisymmetric symbol with unit norm $\left|\varepsilon^{\mu \nu \alpha \beta}\right|=1$. 


\section{B. Electric lagrangian variables}

Achterberg 7] developed a variational principle for relativistic magnetohydrodynamics in which the Lagrangian coordinates $x_{0}^{\mu}$ of the fluid are the field variables, moving in a fixed Eulerian space $x^{\mu}$. In the ideal MHD limit, where the electric field vanishes in the rest frame of the fluid, the Lie derivative of the Faraday two-form vanishes, $£_{u} F=0$, along the direction $u$ of fluid motion. One thereby obtains a simple relation between the background (reference) state and the dynamic state of the magnetofluid. Solving for the $x_{0}^{\mu}$ allows one to obtain all relevant information about the system.

The Faraday two-form is related to its background counterpart by

$$
F_{\mu \nu}=\frac{\partial x_{0}^{\alpha}}{\partial x^{\mu}} \frac{\partial x_{0}^{\beta}}{\partial x^{\nu}} F_{\alpha \beta}^{(0)} .
$$

In the force-free limit, the action is

$$
S=-\int d^{4} x \frac{1}{4} \sqrt{-g} g^{\mu \rho} g^{\nu \sigma} F_{\mu \nu} F_{\rho \sigma} .
$$

By extremizing this action, the equation of motion

$$
J^{\mu} F_{\mu \nu}=0
$$

can be obtained. Written in terms of the coordinate fields this is

$$
\frac{\partial x_{0}^{\alpha}}{\partial x^{\mu}} F_{\alpha \nu}^{(0)} \frac{\partial}{\partial x^{\rho}}\left(\sqrt{-g} g^{\rho \sigma} g^{\mu \tau} \frac{\partial x_{0}^{\gamma}}{\partial x^{\sigma}} \frac{\partial x_{0}^{\delta}}{\partial x^{\tau}} F_{\gamma \delta}^{(0)}\right)=0
$$

Here, we have eliminated a factor of $\partial x_{0}^{\beta} / \partial x^{\nu}$ from $F_{\mu \nu}$. The Bianchi identities are automatically satisfied as long as they are satisfied by the background field. Once the equations for these coordinate fields have been solved, the electromagnetic field is obtained from Eq. [6].

\section{Magnetic lagrangian variables}

Two new MHD formalisms in the extreme relativistic limit were recently developed by Thompson and Blaes [3]. The first of these is related by a duality transformation to the action presented by Achterberg, and is of interest here. In this formalism, the dual tensor $\widetilde{F}$ (rather than $F$ ) plays a central role and the dynamical fields are the perturbed coordinates $x^{\mu}$ (rather than the $x_{0}^{\mu}$ ). In this section, we generalize the treatment of [3] to include gravity.

Once the fields $x^{\mu}$ are solved, the electromagnetic fields can be found from the transformation

$$
\widetilde{F}^{\mu \nu}=\frac{\sqrt{-g\left(x_{0}\right)}}{J_{4} \sqrt{-g(x)}} \frac{\partial x^{\mu}}{\partial x_{0}^{\alpha}} \frac{\partial x^{\nu}}{\partial x_{0}^{\beta}} \widetilde{F}_{0}^{\alpha \beta}
$$

Here

$$
J_{4}=\operatorname{det}\left(\frac{\partial x^{\mu}}{\partial x_{0}^{\alpha}}\right)
$$

is the Jacobian of the transformation $x_{0}^{\alpha} \rightarrow x^{\mu}$ from Lagrangian to Eulerian variables, and the factor of $\sqrt{g_{0} / g}$ is necessary in a curvilinear coordinate system. We show in Appendix A that the force-free equation $J^{\mu} F_{\mu \nu}=0$ may be derived from the action

$$
S^{\prime}=\int d^{4} x_{0} L^{\prime}=\int d^{4} x \frac{1}{4} \sqrt{-g} g_{\mu \rho} g_{\nu \sigma} \widetilde{F}^{\mu \nu} \widetilde{F}^{\rho \sigma}=\int d^{4} x_{0} \frac{J_{4}}{4} \sqrt{-g} g_{\mu \rho} g_{\nu \sigma} \widetilde{F}^{\mu \nu} \tilde{F}^{\rho \sigma} .
$$

Along the way, we obtain the equation of motion

$$
\widetilde{F}^{\delta \varepsilon} \frac{\partial}{\partial x^{\varepsilon}}\left[g_{\beta \delta} g_{\gamma \mu} \widetilde{F}^{\beta \gamma}\right]-\frac{1}{2} \widetilde{F}^{\delta \varepsilon} \frac{\partial}{\partial x^{\mu}}\left[g_{\beta \delta} g_{\gamma \varepsilon} \widetilde{F}^{\beta \gamma}\right]=0 .
$$


In cases where the background magnetic field asymptotes to a constant at large distances, we can use the simple background

$$
\widetilde{F}_{0}^{\mu \nu}=B_{0}\left(\delta_{0}^{\mu} \delta_{3}^{\nu}-\delta_{3}^{\mu} \delta_{0}^{\nu}\right) ; \quad \sqrt{-g_{0}}=1
$$

and define proper time $\tau \equiv t_{0}$ and distance $\sigma \equiv z_{0}$. The equation of motion (13) then takes the form

$$
\frac{\partial x^{\varepsilon}}{\partial x_{0}^{\alpha}}\left(\frac{\partial x^{\delta}}{\partial \tau} \frac{\partial}{\partial \sigma}-\frac{\partial x^{\delta}}{\partial \sigma} \frac{\partial}{\partial \tau}\right) \Sigma_{\delta \varepsilon}-\left(\frac{\partial x^{\delta}}{\partial \tau} \frac{\partial x^{\varepsilon}}{\partial \sigma}\right) \frac{\partial}{\partial x_{0}^{\alpha}} \Sigma_{\delta \varepsilon}=0
$$

where

$$
\Sigma_{\mu \nu} \equiv \frac{g_{\mu \alpha} g_{\nu \beta}}{\sqrt{-g} J_{4}}\left(\frac{\partial x^{\alpha}}{\partial \tau} \frac{\partial x^{\beta}}{\partial \sigma}-\frac{\partial x^{\beta}}{\partial \tau} \frac{\partial x^{\alpha}}{\partial \sigma}\right)=-\Sigma_{\nu \mu}
$$

Although Eq. (13) has four components, it is straightforward to check that the longitudinal components $\mu=0,3$ vanish. Physically, this is equivalent to the statement that MHD waves have two independent transverse polarizations. Notice also that it is possible to simplify Eq. (13) further by choosing a time slicing in which

$$
\sqrt{-g} J_{4}=1
$$

The cost of this gauge choice is that one can no longer identify the background time coordinate $t$ with the proper time $\tau$ of the magnetofluid.

\section{Uniform magnetic field: Spherically symmetric spacetime}

There is a simple prescription for constructing a uniform magnetic field $\left(J^{\phi}=0\right)$ in a vacuum spacetime with an axial killing vector $\xi^{\mu}=g_{\phi}^{\mu}$. One identifies [8]

$$
A_{\mu}=\frac{1}{2} B_{0} \xi_{\mu}=\frac{1}{2} B_{0} g_{\phi \mu} .
$$

Thus $F_{\mu \nu}$ takes exactly the same form outside a black hole of vanishing charge and spin, in the standard Schwarzschild coordinates $\left(g_{\phi \phi}=r^{2} \sin ^{2} \theta\right)$, as it does in Minkowski space:

$$
F_{r \phi}=B_{0} r \sin ^{2} \theta ; \quad F_{\theta \phi}=B_{0} r^{2} \sin \theta \cos \theta .
$$

More generally this method is of limited utility, since it depends on the vanishing of the Ricci tensor of the background spacetime. To give a further illustration of the lagrangian fluid method, we show how it may be used to construct a uniform magnetic field in a general spherical spacetime. The metric may be written as

$$
d s^{2}=g_{t t} d t^{2}+g_{r r} d r^{2}+r^{2}\left(d \theta^{2}+\sin ^{2} \theta d \phi^{2}\right)
$$

We imagine that the currents supporting the magnetic field are flowing far outside the self-gravitating mass, which itself is not electrically conducting. Then

$$
F_{\mu \nu}=\left(\partial_{\mu} R_{0} \partial_{\nu} \phi_{0}-\partial_{\mu} \phi_{0} \partial_{\nu} R_{0}\right) F_{R \phi}^{0}=\frac{1}{2}\left(\partial_{\mu} R_{0}^{2} \partial_{\nu} \phi-\partial_{\mu} \phi_{0} \partial_{\nu} R_{0}^{2}\right) B_{0}
$$

Here $R_{0}=r_{0} \sin \theta_{0}$ is the cylindrical radius and $F_{R \phi}^{0}=B_{0} R_{0}$ is the field strength that would be supported by the same currents in flat space. The vanishing of

$$
4 \pi \sqrt{-g} J^{\phi}=\partial_{r}\left(\sqrt{-g} g^{\phi \phi} g^{r r} F_{\phi r}\right)+\partial_{\theta}\left(\sqrt{-g} g^{\phi \phi} g^{\theta \theta} F_{\phi \theta}\right)
$$

combined with the separation of variables

$$
r_{0}^{2} \sin ^{2} \theta_{0}=\mathcal{R}(r) \mathcal{T}(\theta)
$$

gives the coupled ordinary differential equations

$$
\frac{r^{2}}{\mathcal{R} \sqrt{-g_{t t} g_{r r}}} \frac{d}{d r}\left(\sqrt{-\frac{g_{t t}}{g_{r r}}} \frac{d \mathcal{R}}{d r}\right)=-\frac{\sin \theta}{\mathcal{T}} \frac{d}{d \theta}\left(\frac{1}{\sin \theta} \frac{d \mathcal{T}}{d \theta}\right) .
$$


Because the equation for $\mathcal{T}$ is the same as in flat space, we may write

$$
\sin \theta_{0}=\sin \theta
$$

and

$$
F_{r \phi}=\frac{1}{2} B_{0} \frac{d \mathcal{R}}{d r} \sin ^{2} \theta ; \quad F_{\theta \phi}=B_{0} \mathcal{R} \sin \theta \cos \theta
$$

where $\mathcal{R}$ is a solution to

$$
r^{2} \frac{d}{d r}\left(\sqrt{\frac{-g_{t t}}{g_{r r}}} \frac{d \mathcal{R}}{d r}\right)-2 \sqrt{-g_{t t} g_{r r}} \mathcal{R}=0 .
$$

If the Ricci tensor of the background spacetime vanishes at large radius, then the appropriate solution to (27) must match onto $\mathcal{R}(r)=r^{2}$ at large $r$ (where $\left.-g_{t t}=g_{r r}^{-1}=1-2 G M / r\right)$.

\section{E. Uniform electromagnetic field: Cylindrically symmetric spacetime} form

We will be concerned with the propagation of MHD waves in cylindrically symmetric geometries of the following

$$
d s^{2}=g_{t t} d t^{2}+g_{r r} d r^{2}+g_{\phi \phi} d \phi^{2}+g_{z z} d z^{2}+2 g_{t \phi} d t d \phi
$$

When $g_{t \phi} \neq 0$, the frame dragging of the background $z$-magnetic field generates a radial electric field. Thus, the background electromagnetic field is a stationary, axisymmetric, and $z$-independent solution to the equation $J^{\mu}=0$ with non-vanishing components $F_{r \phi}^{(0)}$ and $F_{r t}^{(0)}$,

$$
\begin{aligned}
& \partial_{r}\left(\sqrt{-g} g^{t t} g^{r r} F_{t r}^{(0)}+\sqrt{-g} g^{t \phi} g^{r r} F_{\phi r}^{(0)}\right)=0 \\
& \partial_{r}\left(\sqrt{-g} g^{\phi \phi} g^{r r} F_{\phi r}^{(0)}+\sqrt{-g} g^{\phi t} g^{r r} F_{t r}^{(0)}\right)=0 .
\end{aligned}
$$

Since the electric field vanishes in the static limit, one has

$$
F_{r t}^{(0)}=-\frac{g_{0}^{t \phi}}{g_{0}^{t t}} F_{r \phi}^{(0)}
$$

and

$$
F_{r \phi}^{(0)}=\frac{B_{0}}{\sqrt{-g_{0}} g_{0}^{r r}\left[g_{0}^{\phi \phi}-\left(g_{0}^{t \phi}\right)^{2} / g_{0}^{t t}\right]}=\frac{g_{0 r r} g_{0 \phi \phi}}{\sqrt{-g_{0}}} B_{0}
$$

[Here $g_{0} \equiv g\left(x_{0}\right)$.]

We will investigate perturbations to the fields, subject to the constraint $F^{\mu \nu} \widetilde{F}_{\mu \nu}=0$, in spacetimes with varying degrees of curvature. It is useful first to consider the case of a nearly flat and non-rotating cylindrical spacetime. In general, static spacetimes which are symmetric about the z-axis may be written in following form [9]

$$
d s^{2}=-e^{2 \lambda} d t^{2}+e^{2(\nu-\lambda)}\left(d r^{2}+d z^{2}\right)+r^{2} e^{-2 \lambda} d \phi^{2}
$$

The function $\lambda$ satisfies Laplace's equation in cylindrical coordinates, and $\nu$ is second order in $\lambda$. In the weak field limit this metric reduces to

$$
d s^{2}=-(1+2 \lambda) d t^{2}+(1-2 \lambda)\left(d r^{2}+d z^{2}\right)+r^{2}(1-2 \lambda) d \phi^{2}
$$

and

$$
\lambda=-G \int d^{3} x^{\prime} \frac{\rho\left(x^{\prime}\right)}{\left|x-x^{\prime}\right|}
$$


becomes the usual Newtonian potential. The background fields (32) and (31) now reduce to

$$
F_{r \phi}^{(0)}=(1-2 \lambda) B_{0} r ; \quad F_{r t}^{(0)}=0 .
$$

A cylindrically symmetric spacetime with some attributes of $3+1$-dimensional black holes can be constructed from the product of $2+1$-dimensional black hole (sitting in the $r$ - $\phi$ plane) and an infinite line segment along the $z$-axis. The $2+1$ black hole solution was originally found by Banados, Teitelboim and Zanelli [10]; its properties are reviewed by Carlip [11]. The key element in its construction is the introduction of a negative cosmological constant $\Lambda=-\frac{1}{\ell^{2}}$, so that the spacetime asymptotes to anti-de Sitter space at large radius. The BTZ metric is

$$
d s^{2}=-\left(-M+\frac{r^{2}}{\ell^{2}}+\frac{J^{2}}{4 r^{2}}\right) d t^{2}+\left(-M+\frac{r^{2}}{\ell^{2}}+\frac{J^{2}}{4 r^{2}}\right)^{-1} d r^{2}+r^{2}\left(d \phi-\frac{J}{2 r^{2}} d t\right)^{2}+d z^{2},
$$

or equivalently

$$
d s^{2}=-(Z R) d t^{2}+(Z R)^{-1} d r^{2}+r^{2}\left(d \phi-\frac{J}{2 r^{2}} d t\right)^{2}+d z^{2}
$$

where

$$
Z \equiv-M+\frac{r^{2}}{\ell^{2}}
$$

and

$$
R \equiv 1-\frac{g_{t \phi}^{2}}{g_{t t} g_{\phi \phi}}=1+\frac{J^{2}}{4 Z r^{2}}
$$

(The variable $M$, being a mass per unit length, is dimensionless in units where $G=c=1$.) The background Maxwell fields in this spacetime are

$$
\begin{gathered}
F_{r \phi}^{(0)}=\frac{B_{0} r}{Z R} ; \\
F_{r t}^{(0)}=\frac{J}{2 r^{2}} F_{r \phi}^{(0)}=\left(\frac{J}{2 r^{2}}\right) \frac{B_{0} r}{Z R} .
\end{gathered}
$$

These expressions reduce to

$$
F_{r \phi}^{(0)}=\frac{B_{0} r}{r^{2} / \ell^{2}-M} ; \quad F_{r t}^{(0)}=0
$$

in the static limit $(J=0)$. Minkowski space is recovered by taking $M \rightarrow-1$ and $\ell^{2} \rightarrow \infty$.

\section{FORCE-FREE MHD WAVES IN CURVED SPACETIMES}

\section{A. MHD modes: Force-free limit}

We first review the normal modes of a uniform magnetofluid in flat Minkowski space, before considering their interactions and the effects of spacetime curvature. The background magnetic field is $\mathbf{B}=B_{0} \hat{z}$, or equivalently $F_{r \phi}=B_{0} r$ in cylindrical coordinates. The fluid supports two distinct modes in the force-free limit: the fast mode, which involves a compressive disturbance and has an isotropic dispersion relation $\omega=|\mathbf{k}|$; and the Alfvén mode, which is incompressible and has a group velocity directed along the background magnetic field, $d \omega / d k_{z}= \pm 1$. There is no slow mode.

The simplest way of seeing that the fluid supports only two normal modes is to note that the perturbation to the Faraday tensor

$$
\delta F_{\mu \nu}=F_{\mu \nu}-F_{\mu \nu}^{(0)},
$$


can be expressed in terms of two variables $\delta r$ and $\delta \phi$. To first order in the perturbation, we have

$$
\delta F_{r \phi}^{(1)}=\frac{\partial}{\partial r}\left(F_{r \phi}^{(0)} \delta r\right)+\frac{\partial \delta \phi}{\partial \phi} F_{r \phi}^{(0)} ; \quad \delta F_{t \phi}^{(1)}=\frac{\partial \delta r}{\partial t} F_{r \phi}^{(0)} ; \quad \delta F_{z \phi}^{(1)}=\frac{\partial \delta r}{\partial z} F_{r \phi}^{(0)},
$$

and

$$
\delta F_{t r}^{(1)}=-\frac{\partial \delta \phi}{\partial t} F_{r \phi}^{(0)} ; \quad \delta F_{z r}^{(1)}=-\frac{\partial \delta \phi}{\partial z} F_{r \phi}^{(0)} ; \quad \delta F_{t z}^{(1)}=0 .
$$

As expected, the component of the electric field parallel to $\mathbf{B}_{0}$ vanishes to linear order. The absence of the slow mode can be related to the invariance of $F_{\mu \nu}^{(0)}$ under reparameterizations of the $z$-coordinate. The full expressions for $\delta F_{\mu \nu}$, written in terms of the Lagrangian fluid variables, are collected in Appendix A.

More generally, the Alfvén mode and fast mode involve perturbations to both fluid coordinates. These perturbations are related by the condition of incompressibility

$$
\frac{1}{r} \frac{\partial(r \delta r)}{\partial r}+\frac{\partial \delta \phi}{\partial \phi}=0 \quad \text { (Alfven) }
$$

in the case of the Alfvén mode; and the condition of vanishing torsion

$$
\frac{\partial \delta r}{\partial \phi}-\frac{\partial\left(r^{2} \delta \phi\right)}{\partial r}=0 \quad(\text { Fast })
$$

in the case of the fast mode. The fluctuating current associated with the field perturbations (45), (46) is

$$
\begin{gathered}
4 \pi \sqrt{-g} J^{t(1)}=\partial_{r}\left(\sqrt{-g} g^{t t} g^{r r} \delta F_{t r}^{(1)}\right)+\partial_{\phi}\left(\sqrt{-g} g^{t t} g^{\phi \phi} \delta F_{t \phi}^{(1)}\right) ; \\
4 \pi \sqrt{-g} J^{z(1)}=\partial_{r}\left(\sqrt{-g} g^{z z} g^{r r} \delta F_{z r}^{(1)}\right)+\partial_{\phi}\left(\sqrt{-g} g^{z z} g^{\phi \phi} \delta F_{z \phi}^{(1)}\right) ; \\
4 \pi \sqrt{-g} J^{r(1)}=\partial_{t}\left(\sqrt{-g} g^{r r} g^{t t} \delta F_{r t}^{(1)}\right)+\partial_{z}\left(\sqrt{-g} g^{r r} g^{z z} \delta F_{r z}^{(1)}\right)+\partial_{\phi}\left(\sqrt{-g} g^{r r} g^{\phi \phi} \delta F_{r \phi}^{(1)}\right) ;
\end{gathered}
$$

and

$$
4 \pi \sqrt{-g} J^{\phi(1)}=\partial_{r}\left(\sqrt{-g} g^{\phi \phi} g^{r r} \delta F_{\phi r}^{(1)}\right)+\partial_{t}\left(\sqrt{-g} g^{\phi \phi} g^{t t} \delta F_{\phi t}^{(1)}\right)+\partial_{z}\left(\sqrt{-g} g^{\phi \phi} g^{z z} \delta F_{\phi z}^{(1)}\right)
$$

Expressed in terms of the coordinate perturbations, and specializing to Minkowski space, this becomes

$$
\begin{gathered}
4 \pi r J^{t(1)}=B_{0} \partial_{t}\left[\partial_{r}\left(r^{2} \delta \phi\right)-\partial_{\phi} \delta r\right] \\
4 \pi r J^{z(1)}=-B_{0} \partial_{z}\left[\partial_{r}\left(r^{2} \delta \phi\right)-\partial_{\phi} \delta r\right] \\
4 \pi r J^{r(1)}=B_{0} r^{2}\left\{-\partial_{t}^{2} \delta \phi+\partial_{z}^{2} \delta \phi+\frac{1}{r^{2}} \partial_{\phi}\left[\frac{1}{r} \partial_{r}(r \delta r)+\partial_{\phi} \delta \phi\right]\right\}
\end{gathered}
$$

and

$$
4 \pi r J^{\phi(1)}=-B_{0}\left\{-\partial_{t}^{2} \delta r+\partial_{z}^{2} \delta r+\partial_{r}\left[\frac{1}{r} \partial_{r}(r \delta r)+\partial_{\phi} \delta \phi\right]\right\} .
$$

The dynamical equations for the two normal modes are recovered by imposing the force-free condition. To linear order one has

$$
J^{r(1)} F_{r \phi}^{(0)}=0=J^{\phi(1)} F_{\phi r}^{(0)}
$$


hence $J^{r(1)}=J^{\phi(1)}=0$. In the case of the Alfvén mode, applying the constraint (47) gives the one-dimensional wave equation

$$
\begin{aligned}
-\partial_{t}^{2} \delta r+\partial_{z}^{2} \delta r & =0 \\
-\partial_{t}^{2} \delta \phi+\partial_{z}^{2} \delta \phi & =0
\end{aligned}
$$

with a vanishing longitudinal field perturbation

$$
\delta F_{r \phi}^{(1)}=0
$$

and (in general) a non-vanishing longitudinal current

$$
J^{t(1)}, J^{z(1)} \neq 0
$$

A force-free magnetofluid supports torsional waves of both helicities. It should be noted that, in this limit, there is no basic distinction between the two helicities, as there is a non-relativistic cold, magnetized plasma.

In the case of the fast mode, one instead finds that the current vanishes entirely and the coordinate perturbations obey three-dimensional wave equations,

$$
\begin{aligned}
-\partial_{t}^{2} \delta \phi+\partial_{z}^{2} \delta \phi+\frac{1}{r^{3}} \partial_{r}\left[r \partial_{r}\left(r^{2} \delta \phi\right)\right]+\frac{1}{r^{2}} \partial_{\phi}^{2} \delta \phi & =0 \\
-\partial_{t}^{2} \delta r+\partial_{z}^{2} \delta r+\partial_{r}\left[\frac{1}{r} \partial_{r}(r \delta r)\right]+\frac{1}{r^{2}} \partial_{\phi}^{2} \delta r & =\frac{2}{r} \partial_{\phi} \delta \phi
\end{aligned}
$$

By applying the operator $r^{-1} \partial_{r} r$ to the first equation, one sees that an axisymmetric field perturbation

$$
\frac{\delta B^{z}}{B_{0}}=\frac{\delta F_{\phi r}^{(1)}}{F_{\phi r}^{(0)}}=\frac{1}{r} \frac{\partial}{\partial r}(r \delta r)
$$

satisfies the (axisymmetric) cylindrical wave equation.

Thus, the fast mode is equivalent to a vacuum electromagnetic wave superposed on the uniform background magnetic field, but with its polarization restricted to $\delta \mathbf{E} \cdot \mathbf{B}_{0}=0$. This way of constructing the fast mode also makes it immediately apparent that infinite plane waves (restricted to this single polarization state) are another general class of solutions.

Inspection of Eqs. (55) and (56) shows that another projection,

$$
\frac{1}{r^{2}} \frac{\partial \delta r}{\partial \phi}-\frac{\partial \delta \phi}{\partial r}=0
$$

allows the equations of motion for $\delta r$ and $\delta \phi$ to be entirely separated,

$$
\begin{array}{r}
-\partial_{t}^{2} \delta \phi+\partial_{z}^{2} \delta \phi+\frac{1}{r^{3}} \partial_{r}\left(r^{3} \partial_{r} \delta \phi\right)+\frac{1}{r^{2}} \partial_{\phi}^{2} \delta \phi=0 \\
-\partial_{t}^{2} \delta r+\partial_{z}^{2} \delta r+\partial_{r}\left[\frac{1}{r} \partial_{r}(r \delta r)\right]+\frac{1}{r^{2}} \partial_{\phi}^{2} \delta r=0 .
\end{array}
$$

At the same time, the longitudinal currents do not vanish,

$$
J^{t(1)}=\frac{B_{0}}{2 \pi} \partial_{t} \delta \phi ; \quad J^{z(1)}=-\frac{B_{0}}{2 \pi} \partial_{z} \delta \phi .
$$

\section{B. Decomposition into normal modes in a curved spacetime}

We have seen that, even in Minkowski space, there is an ambiguity in the definition of the fast mode. Axially symmetric perturbations decompose directly into torsional modes, which are supported only by a perturbation $\delta \phi$, and compressive modes, which are supported only by a perturbation $\delta r$. In the more general case of non-axisymmetric perturbations, one can still define unambiguously an incompressible mode (47). But one is faced with a choice either of defining the fast mode to be current free through Eq. (48), in which case the equations of motion (61) for $\delta r$ and 
$\delta \phi$ do not entirely separate; or instead of using the alternative projection (63) which separates these equations but leaves a non-vanishing longitudinal current [65].

This ambiguity remains when one considers the case of a magnetofluid in a gravitational field. We focus here on a general static $\left(g_{t \phi}=0\right)$ and cylindrically symmetric spacetime. In this case, the longitudinal currents (49) and (50) cannot both vanish, as is seen by writing them in terms of the coordinate perturbations:

$$
\frac{4 \pi}{B_{0}} \sqrt{-g} J^{t(1)}=\frac{\partial}{\partial t}\left[-\frac{\partial}{\partial r}\left(\frac{g_{\phi \phi}}{g_{t t}} \delta \phi\right)+\frac{g_{r r}}{g_{t t}} \frac{\partial \delta r}{\partial \phi}\right]
$$

and

$$
\frac{4 \pi}{B_{0}} \sqrt{-g} J^{z(1)}=\frac{\partial}{\partial z}\left[-\frac{\partial}{\partial r}\left(\frac{g_{\phi \phi}}{g_{z z}} \delta \phi\right)+\frac{g_{r r}}{g_{z z}} \frac{\partial \delta r}{\partial \phi}\right] .
$$

In these expressions, $g_{t t}$ and $g_{z z}$ have different dependences on radius $r$, and we have made use of the diagonality of the metric. We conclude that, to linear order in the field perturbation, non-axisymmetric excitations of the fast mode are current-carrying.

It is, nonetheless, possible to choose a generalization of the projection (63) which does allow the equations of motion for $\delta r$ and $\delta \phi$ to decouple at linear order. From Eqs. (66) and (67), the obvious choice is

$$
\frac{g_{r r}}{g_{\phi \phi}} \frac{\partial \delta r}{\partial \phi}-\frac{\partial \delta \phi}{\partial r}=0 . \quad \text { (Fast) }
$$

Then Eq. (52) becomes

$$
\left(\frac{1}{g_{t t}} \frac{\partial^{2} \delta r}{\partial t^{2}}+\frac{1}{g_{z z}} \frac{\partial^{2} \delta r}{\partial z^{2}}\right) F_{r \phi}^{(0)}+\frac{1}{g_{\phi \phi}} \frac{\partial^{2} \delta r}{\partial \phi^{2}} F_{r \phi}^{(0)}+\frac{g_{\phi \phi}}{\sqrt{-g}} \frac{\partial}{\partial r}\left[\frac{\sqrt{-g}}{g_{\phi \phi} g_{r r}} \frac{\partial}{\partial r}\left(\delta r F_{r \phi}^{(0)}\right)\right]=0,
$$

or, upon substituting expression (32),

$$
\frac{1}{g_{t t}} \frac{\partial^{2} \delta r}{\partial t^{2}}+\frac{1}{g_{z z}} \frac{\partial^{2} \delta r}{\partial z^{2}}+\frac{1}{g_{\phi \phi}} \frac{\partial^{2} \delta r}{\partial \phi^{2}}+\frac{1}{g_{r r}} \frac{\partial}{\partial r}\left[\frac{\sqrt{-g}}{g_{\phi \phi} g_{r r}} \frac{\partial}{\partial r}\left(\delta r \frac{g_{r r} g_{\phi \phi}}{\sqrt{-g}}\right)\right]=0 .
$$

The longitudinal currents then are

$$
\frac{4 \pi}{B_{0}} \sqrt{-g} J^{t(1)}=-\frac{\partial}{\partial r}\left(\frac{g_{\phi \phi}}{g_{t t}}\right) \frac{\partial \delta \phi}{\partial t} ; \quad \frac{4 \pi}{B_{0}} \sqrt{-g} J^{z(1)}=-\frac{\partial}{\partial r}\left(\frac{g_{\phi \phi}}{g_{z z}}\right) \frac{\partial \delta \phi}{\partial z} .
$$

The perturbation $\delta \phi$ can be recovered from $\delta r$ using the constraint [68). Similarly, the incompressible Alfvén mode is defined by

$$
\frac{\sqrt{-g}}{g_{r r} g_{\phi \phi}} \frac{\partial}{\partial r}\left(\frac{g_{r r} g_{\phi \phi}}{\sqrt{-g}} \delta r\right)+\frac{\partial \delta \phi}{\partial \phi}=0, \quad \text { (Alfven) }
$$

to linear order.

For example, the wave equation for a purely compressive disturbance in a BTZ black string spacetime is

$$
-\frac{1}{Z^{2}} \frac{\partial^{2} \delta r}{\partial t^{2}}+\frac{1}{Z} \frac{\partial^{2} \delta r}{\partial z^{2}}+\frac{1}{r^{2} Z} \frac{\partial^{2} \delta r}{\partial \phi^{2}}+\frac{\partial}{\partial r}\left[\frac{Z}{r} \frac{\partial}{\partial r}\left(\frac{r \delta r}{Z}\right)\right]=0 .
$$

(As before, $Z(r) \equiv r^{2} / \ell^{2}-M$.) When $\partial_{z} \delta r=\partial_{\phi} \delta r=0$, this equation can also be re-expressed directly in terms of the field perturbation

$$
\frac{\delta B^{z}}{B^{z}}=\frac{Z}{r} \frac{\partial}{\partial r}\left(\frac{r \delta r}{Z}\right)
$$

as

$$
-\frac{\partial^{2}}{\partial t^{2}}\left(\frac{\delta B^{z}}{B^{z}}\right)+\frac{Z}{r} \frac{\partial}{\partial r}\left[Z r \frac{\partial}{\partial r}\left(\frac{\delta B^{z}}{B^{z}}\right)\right]=0
$$

More generally, the field perturbations have some dependence on $z$, and it is more useful to work with the coordinate perturbation. 


\section{SCATTERING OF TORSIONAL WAVES BY SPACETIME CURVATURE}

We now turn to an important, but subtle, difference between the compressive (fast) and torsional (Alfvén) modes in a curved spacetime. We focus on a uniform magnetofluid in which the background current vanishes. Both modes are exact non-linear solutions to the force-free equation in Minkowski space. This property is retained by a purely radial oscillation $(\delta \phi=0)$, in any static cylindrically symmetric (or spherically symmetric) spacetime. The resulting fast mode is equivalent to an electromagnetic wave polarized in the $\phi$-direction, with $\delta \mathbf{E} \cdot \mathbf{B}_{0}=0$. Hence it can have arbitrary amplitude.

An axisymmetric torsional wave is not, by contrast, a non-linear solution to $J^{\mu} F_{\mu \nu}=0$, because the wave speed varies with radius. We now show that, in a static spacetime, such an Alfvén wave will excite a compressive motion transverse to the background magnetic field. The amplitude of this compressive motion is second order in the amplitude $\varepsilon$ of the Alfvén wave itself. This effect is somewhat analogous to the non-linear coupling between a fast mode and an Alfvén wave that is propagating through a non-relativistic magnetofluid with a gradient in density and in Alfvén speed (e.g. 12], 13]). In a spherical spacetime, we show that a torsional wave originating at infinity is scattered into a spherical outgoing compressive wave.

To this end, we expand the field and currents perturbations in powers of $\varepsilon \ll 1$ :

$$
\begin{gathered}
F_{\mu \nu}=F_{\mu \nu}^{(0)}+\delta F_{\mu \nu}^{(1)}+\delta F_{\mu \nu}^{(2)}+O\left(\varepsilon^{3}\right) ; \\
J^{\mu}=\delta J^{\mu(1)}+\delta J^{\mu(2)}+O\left(\varepsilon^{3}\right) .
\end{gathered}
$$

Here, $\delta F_{\mu \nu}^{(N)}=O\left(\varepsilon^{N}\right)$, etc.

When considering the effects of spacetime curvature, we also restrict ourselves to axisymmetric perturbations. In a static spacetime, this means that $\delta r$ and $\delta \phi$ decouple to linear order. Such a linear coupling is re-established in a rotating spacetime.

\section{A. Torsional waves in a cylindrically symmetric spacetime}

$$
\text { 1. Static spacetime }\left(g_{t \phi}=0\right)
$$

Torsional deformations of the magnetic field are supported by electric currents which, to leading order in $\varepsilon$, propagate along the background magnetic field. We first derive the analog, in a curved cylindrical spacetime, of the linear wave equation (58). With this in hand, we find solutions to $J^{\mu} F_{\mu \nu}=0$ to second order in $\varepsilon$.

The force-free equation implies $J^{r(1)}=0$ to linear order (Eq. [57). Combining expression (51) with the with the Bianchi identity $\partial_{t} \delta F_{r z}^{(1)}=\partial_{z} \delta F_{r t}^{(1)}$ gives

$$
g^{t t} \partial_{t}^{2}\left(\delta F_{r z}^{(1)}\right)+g^{z z} \partial_{z}^{2}\left(\delta F_{r z}^{(1)}\right)=0
$$

The electric field $\delta F_{r t}^{(1)}$ satisfies the same wave equation. The solution is

$$
\delta F_{r z}^{ \pm}=\delta F_{r z}^{ \pm}\left(z \mp v_{z} t\right) ; \quad \delta F_{r t}^{ \pm}=\delta F_{r t}^{ \pm}\left(z \mp v_{z} t\right) .
$$

where

$$
v_{z} \equiv \sqrt{-\frac{g_{t t}}{g_{z z}}} .
$$

The two fluctuating field components are related by

$$
\delta F_{t r}^{ \pm}=\mp v_{z} \delta F_{z r}^{ \pm} .
$$

The net result is that the Alfvén wave propagates along the $z$-axis, to leading order in $\varepsilon$, with a phase speed equal to that of a null geodesic that lies tangent to the background magnetic field.

An Alfvén wave front propagating along the background magnetic field will be bent as the result of the dependence of $g_{t t}$ (and $v_{z}$ ) on radius. This, in turn, forces a radial oscillation of the magnetofluid that is second order in $\varepsilon$. Indeed, inspection of the force-free equation

$$
J^{t(1)} \delta F_{t r}^{(1)}+J^{z(1)} \delta F_{z r}^{(1)}+J^{\phi(2)} F_{\phi r}^{(0)}=0,
$$


shows that the first two terms generally do not cancel. Using Eq. (81) to eliminate $\delta F_{t r}$ in terms of $\delta F_{z r}$, one obtains

$$
g^{r r} g^{z z}\left(\delta F_{z r}^{(1)}\right)^{2} \partial_{r}\left(\ln v_{z}\right)+4 \pi J^{\phi(2)} F_{\phi r}^{(0)}=0 .
$$

The radial fluctuation of the magnetofluid supports the additional current perturbation,

$$
4 \pi \sqrt{-g} J^{\phi(2)}=-B_{0}\left\{\frac{g_{r r}}{g_{t t}} \frac{\partial^{2} \delta r}{\partial t^{2}}+\frac{g_{r r}}{g_{z z}} \frac{\partial^{2} \delta r}{\partial z^{2}}+\frac{\partial}{\partial r}\left[\frac{\sqrt{-g}}{g_{\phi \phi} g_{r r}} \frac{\partial}{\partial r}\left(\delta r \frac{g_{r r} g_{\phi \phi}}{\sqrt{-g}}\right)\right]\right\}
$$

Let us first consider the special case of a nearly flat cylindrical spacetime. In such a background, $v_{z}=1+2 \lambda$ and Eq. (83) reduces to

$$
\frac{1}{2 \pi}\left(\delta F_{z r}^{(1)}\right)^{2} \frac{d \lambda}{d r}+J^{\phi(2)} F_{\phi r}^{(0)}=0
$$

to lowest order in $\lambda$. Re-expressing this equation in terms of the perturbations $\delta r$ and $\delta \phi$ gives

$$
-\frac{\partial^{2} \delta r}{\partial t^{2}}+\frac{\partial}{\partial r}\left[\frac{1}{r} \frac{\partial}{\partial r}(r \delta r)\right]+\frac{\partial^{2} \delta r}{\partial z^{2}}=-2 r^{2} \frac{d \lambda}{d r}\left(\frac{\partial \delta \phi}{\partial z}\right)^{2} .
$$

The analogous expressions for the BTZ black string spacetime are not much more complicated. In this case, $v_{z}=Z^{1 / 2}=\left(r^{2} / \ell^{2}-M\right)^{1 / 2}$, which vanishes at the horizon $r_{H}=M^{1 / 2} \ell$. The force-free equation reduces to

$$
\frac{1}{2}\left(\delta F_{z r}^{(1)}\right)^{2} \partial_{r} Z+4 \pi J^{\phi(2)} F_{\phi r}^{(0)}=0
$$

We then have

$$
-\frac{1}{Z^{2}} \frac{\partial^{2} \delta r}{\partial t^{2}}+\frac{1}{Z} \frac{\partial^{2} \delta r}{\partial z^{2}}+\frac{\partial}{\partial r}\left[\frac{Z}{r} \frac{\partial}{\partial r}\left(\frac{r \delta r}{Z}\right)\right]=-\frac{1}{Z} \frac{r^{3}}{l^{2}}\left(\frac{\partial \delta \phi}{\partial z}\right)^{2}
$$

\section{Non-static spacetime $\left(g_{t \phi} \neq 0\right)$}

When the spacetime rotates $\left(g_{t \phi} \neq 0\right)$, one finds that the Alfvén wave ansatz (81) fails to satisfy the force-free equation to linear order in $\varepsilon$. Physically, this is because the torsional and radial modes of the magnetofluid are coupled through conservation of angular momentum. One must therefore introduce simultaneous perturbations in $\delta r$, $\delta \phi$, and $\delta t$. In terms of these variables, the field perturbations become (to first order)

$$
\begin{gathered}
\delta F_{r \phi}^{(1)}=\frac{\partial}{\partial r}\left(F_{r \phi}^{(0)} \delta r\right) ; \quad \delta F_{t \phi}^{(1)}=\frac{\partial(\delta r)}{\partial t} F_{r \phi}^{(0)} ; \quad \delta F_{z \phi}^{(1)}=\frac{\partial(\delta r)}{\partial z} F_{r \phi}^{(0)} ; \\
\delta F_{r t}^{(1)}=\frac{\partial(\delta \widetilde{\phi})}{\partial t} F_{r \phi}^{(0)}-\frac{\partial}{\partial r}\left(\frac{g^{t \phi}}{g^{t t}} F_{r \phi}^{(0)} \delta r\right) ; \quad \delta F_{r z}^{(1)}=\frac{\partial(\delta \widetilde{\phi})}{\partial z} F_{r \phi}^{(0)} ; \quad \delta F_{t z}^{(1)}=-\frac{\partial \delta r}{\partial z}\left(\frac{g^{t \phi}}{g^{t t}}\right) F_{\phi r}^{(0)} .
\end{gathered}
$$

Here

$$
\delta \widetilde{\phi} \equiv \delta \phi-\left(\frac{g^{t \phi}}{g^{t t}}\right) \delta t=\delta \phi+\left(\frac{g_{t \phi}}{g_{\phi \phi}}\right) \delta t
$$

In this situation, the $t$ - $\phi$ components of the metric are not diagonal: $g^{t t}=g_{\phi \phi} / \operatorname{det}(t, \phi), g^{\phi \phi}=g_{t t} / \operatorname{det}(t, \phi)$, and $g^{t \phi}=-g_{t \phi} / \operatorname{det}(t, \phi)$, where $\operatorname{det}(t, \phi) \equiv g_{t t} g_{\phi \phi}-g_{t \phi}^{2}$.

The force-free equations are, again to linear order,

$$
J^{r(1)} F_{r \phi}^{(0)}=0=J^{r(1)} F_{r t}^{(0)}
$$

or equivalently

$$
J^{r(1)}=0
$$


and

$$
J^{t(1)} F_{t r}^{(0)}+J^{\phi(1)} F_{\phi r}^{(0)}=0 .
$$

Finally, the currents are given by

$$
\begin{gathered}
4 \pi \sqrt{-g} J^{t(1)}=\partial_{r}\left(\sqrt{-g} g^{t t} g^{r r} \delta F_{t r}^{(1)}+\sqrt{-g} g^{t \phi} g^{r r} \delta F_{\phi r}^{(1)}\right) \\
+\partial_{z}\left(\sqrt{-g} g^{t t} g^{z z} \delta F_{t z}^{(1)}\right)+\partial_{z}\left(\sqrt{-g} g^{t \phi} g^{z z} \delta F_{\phi z}^{(1)}\right) ; \\
4 \pi \sqrt{-g} J^{r(1)}=\partial_{t}\left(\sqrt{-g} g^{r r} g^{t \phi} \delta F_{r \phi}^{(1)}+\sqrt{-g} g^{r r} g^{t t} \delta F_{r t}^{(1)}\right)+\partial_{z}\left(\sqrt{-g} g^{r r} g^{z z} \delta F_{r z}^{(1)}\right) ;
\end{gathered}
$$

and

$$
\begin{aligned}
4 \pi \sqrt{-g} J^{\phi(1)} & =\partial_{r}\left(\sqrt{-g} g^{\phi \phi} g^{r r} \delta F_{\phi r}^{(1)}+\sqrt{-g} g^{\phi t} g^{r r} \delta F_{t r}^{(1)}\right)-\partial_{t}\left(\sqrt{-g}\left(g^{t \phi}\right)^{2} \delta F_{\phi t}^{(1)}\right) \\
& +\partial_{t}\left(\sqrt{-g} g^{\phi \phi} g^{t t} \delta F_{\phi t}^{(1)}\right)+\partial_{z}\left(\sqrt{-g} g^{\phi \phi} g^{z z} \delta F_{\phi z}^{(1)}\right)+\partial_{z}\left(\sqrt{-g} g^{\phi t} g^{z z} \delta F_{t z}^{(1)}\right) .
\end{aligned}
$$

Substituting for the field perturbations, Eq. (93) becomes

$$
g^{t t} \frac{\partial^{2}(\delta \widetilde{\phi})}{\partial t^{2}}+g^{z z} \frac{\partial^{2}(\delta \tilde{\phi})}{\partial z^{2}}=-g^{t t} \frac{\partial}{\partial r}\left(\frac{g_{t \phi}}{g_{\phi \phi}}\right) \frac{\partial \delta r}{\partial t} .
$$

One quickly obtains an integral of motion from this equation when the gradient in the $z$-direction vanishes:

$$
\frac{\partial \widetilde{\phi}}{\partial t}+\delta r \frac{\partial}{\partial r}\left(\frac{g_{t \phi}}{g_{\phi \phi}}\right)=0 .
$$

The torsional perturbation $\delta \phi$ is slaved to the compressive perturbation $\delta r$ through conservation of angular momentum. The manipulation of the force-free equation (94) is a bit more complicated; some details are given in Appendix B. The final result is

$$
\begin{aligned}
\left(g^{t t} \frac{\partial^{2} \delta r}{\partial t^{2}}+g^{z z} \frac{\partial^{2} \delta r}{\partial z^{2}}\right) F_{r \phi}^{(0)} & +\frac{g_{\phi \phi}}{\sqrt{-g}} \frac{\partial}{\partial r}\left[\frac{\sqrt{-g}}{g_{\phi \phi} g_{r r}} \frac{\partial}{\partial r}\left(\delta r F_{r \phi}^{(0)}\right)\right] \\
& =\frac{g^{t t} g_{\phi \phi}}{g_{r r}}\left[\frac{\partial \widetilde{\phi}}{\partial t}+\delta r \frac{\partial}{\partial r}\left(\frac{g_{t \phi}}{g_{\phi \phi}}\right)\right] \frac{\partial}{\partial r}\left(\frac{g_{t \phi}}{g_{\phi \phi}}\right) F_{r \phi}^{(0)} .
\end{aligned}
$$

The wave operator on the left-hand side of Eqs. (98) and (100) is now mixed with an additional term involving the other coordinate perturbation. This source term can be treated as a perturbation when the spacetime is slowly rotating $\left(g_{t \phi}^{2} \ll\left|g_{t t} g_{\phi \phi}\right|\right)$. It is also worth noting a simplification which takes place when the perturbations are uniform in $z$ : then the right-hand side of Eq. (100) vanishes and, effectively, the fast mode equation involves only a single coordinate perturbation. That is, the time-evolution of the angular perturbation $\delta \phi$ is fixed by conservation of angular momentum, and does not react back on the equation of motion for $\delta r$. More generally this is not the case, and the dynamics involves two independent fields.

\section{B. Scattering of a cylindrical Alfvén wave in a spherical gravitational field}

A uniform magnetofluid is perturbed by a spherical gravitational field (Section IID). We now consider the perturbation to a torsional Alfvén wave propagating along this background magnetic field. We focus on a weak, spherical gravitational field, and allow for the possibility that the gravitating mass is extended in radius. Then the metric departs from the Schwarzschild metric; we write it as

$$
-g_{t t}=1+\Delta_{t t} ; \quad g_{r r}=1+\Delta_{r r} .
$$

(In this section, as in Section [D $r$ is the spherical radius.) In the weak field limit $\left(\left|\Delta_{t t}\right|,\left|\Delta_{r r}\right| \ll 1\right)$ the radial coordinate of the magnetofluid is perturbed by an amount $\delta r / r=O(\Delta)$. 
The static perturbation $\delta r$ is a solution to the linearized version of Eq. (27),

$$
\frac{d^{2}(r \delta r)}{d r^{2}}-2 \frac{(r \delta r)}{r^{2}}=\frac{1}{2}\left(\Delta_{t t}+\Delta_{r r}\right)-\frac{1}{2} \frac{d}{d r}\left[r\left(\Delta_{t t}-\Delta_{r r}\right)\right]
$$

Notice that the right-hand side of this equation vanishes in the Schwarzschild metric, $\Delta_{r r}=-\Delta_{t t}=2 G M / r$. When the enclosed gravitating mass $M$ is itself a function of radius, Eq. (102) becomes

$$
\frac{d^{2}(r \delta r)}{d r^{2}}-2 \frac{(r \delta r)}{r^{2}}=2 G \frac{d M}{d r}
$$

We expand the background magnetic field $F^{(0)}$, the Alfvén wave $\delta F^{(1)}$, and its current $\delta J^{(1)}$ in powers of $\Delta$,

$$
F^{(0)}=F_{0-\Delta}^{(0)}+F_{1-\Delta}^{(0)}
$$

and

$$
\delta F^{(1)}=\delta F_{0-\Delta}^{(1)}+\delta F_{1-\Delta}^{(1)} ; \quad \delta J^{(1)}=\delta J_{0-\Delta}^{(1)}+\delta J_{1-\Delta}^{(1)},
$$

so that $F_{1-\Delta}^{(0)}, \delta F_{1-\Delta}^{(1)}$ and $\delta J_{1-\Delta}^{(1)}$ are first order in $\delta r$. For example, expanding $\mathcal{R}=r_{0}^{2} \simeq r^{2}+2 r \delta r$ in Eq. (26) for the background field, we have

$$
\left[F_{0-\Delta}^{(0)}\right]_{r \phi}=B_{0} r \sin ^{2} \theta ; \quad\left[F_{0-\Delta}^{(0)}\right]_{\theta \phi}=B_{0} r^{2} \sin \theta \cos \theta
$$

and

$$
\left[F_{1-\Delta}^{(0)}\right]_{r \phi}=B_{0} \frac{d(r \delta r)}{d r} \sin ^{2} \theta ; \quad\left[F_{1-\Delta}^{(0)}\right]_{\theta \phi}=2 B_{0} r \delta r \sin \theta \cos \theta
$$

In the absence of gravity, the torsional wave propagates along the magnetic field. The associated charge and current densities

$$
\begin{aligned}
& \delta \rho=\delta J_{0}(z-t) \exp \left[-\frac{1}{2}\left(\frac{r \sin \theta}{R_{0}}\right)^{2}\right] \\
& \delta \mathbf{J}=\delta J_{0}(z-t) \exp \left[-\frac{1}{2}\left(\frac{r \sin \theta}{R_{0}}\right)^{2}\right] \hat{z}
\end{aligned}
$$

are localized within a cylindrical radius $r \sin \theta \sim R_{0}$.

We will focus on the case where the wave is strongly sheared, i.e., where its frequency is small compared with $R_{0}^{-1}$ :

$$
R_{0}\left|\frac{\delta J_{0}^{\prime}(z-t)}{\delta J_{0}(z-t)}\right| \ll 1
$$

To lowest order in the gravitational potential, the components of the fluctuating current and field are

$$
\left[\delta J_{0-\Delta}^{(1)}\right]^{t}=\delta J ; \quad\left[\delta J_{0-\Delta}^{(1)}\right]^{r}=\cos \theta \delta J ; \quad\left[\delta J_{0-\Delta}^{(1)}\right]^{\theta}=-\frac{1}{r} \sin \theta \delta J
$$

and

$$
\begin{aligned}
& {\left[\delta F_{0-\Delta}^{(1)}\right]_{\theta r}=-\frac{4 \pi \delta J_{0} R_{0}^{2}}{\sin \theta}\left\{1-\exp \left[-\frac{1}{2}\left(\frac{r \sin \theta}{R_{0}}\right)^{2}\right]\right\}} \\
& {\left[\delta F_{0-\Delta}^{(1)}\right]_{t r}=\frac{\sin \theta}{r}\left[\delta F_{0-\Delta}^{(1)}\right]_{\theta r} ; \quad\left[\delta F_{0-\Delta}^{(1)}\right]_{t \theta}=\cos \theta\left[\delta F_{0-\Delta}^{(1)}\right]_{\theta r} .}
\end{aligned}
$$

The static gravitational perturbation to the background magnetic field is known implicitly through the solution to Eq. (102). We next solve for the perturbations $\delta F_{1-\Delta}^{(1)}$ and $\delta J_{1-\Delta}^{(1)}$. The current perturbation is determined from the 
linear component of the force-free equation. Making use of $\left[\delta J_{0-\Delta}^{(1)}\right]^{\mu}\left[F_{0-\Delta}^{(0)}\right]_{\mu \nu}=0$ and neglecting the terms that are second-order in $\Delta$, we have

$$
\left[\delta J_{0-\Delta}^{(1)}\right]^{\mu}\left[F_{1-\Delta}^{(0)}\right]_{\mu \nu}+\left[\delta J_{1-\Delta}^{(1)}\right]^{\mu}\left[F_{0-\Delta}^{(0)}\right]_{\mu \nu}=0 .
$$

The force-free equation (112) may be re-expressed by substituting Eqs. (106), (107) and (110),

$$
\left[\delta J_{1-\Delta}^{(1)}\right]^{r} \sin \theta+\left[\delta J_{1-\Delta}^{(1)}\right]^{\theta} r \cos \theta=-\delta J(r \sin \theta, z, t)\left[r \frac{d(\delta r / r)}{d r}\right] \sin \theta \cos \theta .
$$

In the low frequency regime, the equation of current conservation simplifies to

$$
\frac{1}{r^{2}} \frac{\partial}{\partial r}\left\{r^{2}\left[\delta J_{1-\Delta}^{(1)}\right]^{r}\right\}+\frac{1}{\sin \theta} \frac{\partial}{\partial \theta}\left\{\sin \theta\left[\delta J_{1-\Delta}^{(1)}\right]^{\theta}\right\}=0 .
$$

For the particular choice (108) of incident current density, the solution to the combined equations (113) and (114) is

$$
\left[\delta J_{1-\Delta}^{(1)}\right]^{r}=\left[2 \frac{\delta r}{r} \cos \theta-\frac{r \delta r}{R_{0}^{2}} \cos \theta \sin ^{2} \theta\right] \delta J
$$

and

$$
\left[\delta J_{1-\Delta}^{(1)}\right]^{\theta}=\left[-\frac{1}{r^{2}} \frac{d(r \delta r)}{d r} \sin \theta+\frac{\delta r}{R_{0}^{2}} \sin ^{3} \theta\right] \delta J
$$

Notice that the current $\delta J_{1-\Delta}^{(1)}$ receives a contribution from the metric perturbation as well as from $\delta F_{1-\Delta}^{(1)}$, e.g.

$$
4 \pi\left[\delta J_{1-\Delta}^{(1)}\right]^{r}=\frac{1}{r^{2} \sin \theta} \frac{\partial}{\partial \theta}\left\{\sin \theta\left(g^{r r}-1\right)\left[\delta F_{0-\Delta}^{(1)}\right]_{r \theta}\right\}+\frac{1}{r^{2} \sin \theta} \frac{\partial}{\partial \theta}\left\{\sin \theta\left[\delta F_{1-\Delta}^{(1)}\right]_{r \theta}\right\} .
$$

Here, the metric perturbation is $g^{r r}-1=-2 G M / r$ to lowest order. Thus the current components $\left[\delta J_{1-\Delta}^{(1)}\right]^{r, \theta}$ are sourced by the fluctuating field

$$
\left[\delta F_{1-\Delta}^{(1)}\right]_{\theta r}=-[4 \pi(r \delta r) \sin \theta] \delta J(r \sin \theta, z, t)+\frac{2 G M}{r}\left[\delta F_{0-\Delta}^{(1)}\right]_{\theta r} .
$$

The gravitational perturbation of the charge density, and the electric field which it sources, can be obtained by combining the Bianchi identity

$$
\partial_{\theta}\left[\delta F_{1-\Delta}^{(1)}\right]_{t r}-\partial_{r}\left[\delta F_{1-\Delta}^{(1)}\right]_{t \theta}=\partial_{t}\left[\delta F_{1-\Delta}^{(1)}\right]_{\theta r} \simeq 0
$$

with the MHD condition

$$
\begin{gathered}
\left\{\left[\delta F_{0-\Delta}^{(1)}\right]_{t \theta}+\left[\delta F_{1-\Delta}^{(1)}\right]_{t \theta}\right\}\left\{\left[F_{0-\Delta}^{(0)}\right]_{\phi r}+\left[F_{1-\Delta}^{(0)}\right]_{\phi r}\right\} \\
-\left\{\left[\delta F_{0-\Delta}^{(1)}\right]_{t r}+\left[\delta F_{1-\Delta}^{(1)}\right]_{t r}\right\}\left\{\left[F_{0-\Delta}^{(0)}\right]_{\phi \theta}+\left[F_{1-\Delta}^{(0)}\right]_{\phi \theta}\right\}=0 .
\end{gathered}
$$

At leading order in the gravitational potential, this last equation becomes

$$
\left[\delta F_{1-\Delta}^{(1)}\right]_{t \theta}=r \cot \theta\left[\delta F_{1-\Delta}^{(1)}\right]_{t r}-\cos \theta \frac{d\left(r^{3} \delta r\right)}{d \ln r}\left[\delta F_{0-\Delta}^{(1)}\right]_{\theta r} .
$$

Substituting this expression into Eq. (119), one can solve for the perturbation to the electric field,

$$
\left[\delta F_{1-\Delta}^{(1)}\right]_{t r}=-4 \pi \delta J_{0}\left[R_{0}^{2} \frac{d(\delta r / r)}{d r}\left(1-e^{-R^{2} / 2 R_{0}^{2}}\right)+\sin ^{2} \theta \delta r e^{-R^{2} / 2 R_{0}^{2}}\right],
$$

and

$$
\left[\delta F_{1-\Delta}^{(1)}\right]_{t \theta}=-[4 \pi(r \delta r) \sin \theta \cos \theta] \delta J_{0} e^{-R^{2} / 2 R_{0}^{2}}
$$


Here $R=r \sin \theta$. The charge density perturbation is then

$$
\begin{aligned}
4 \pi\left[\delta J_{1-\Delta}^{(1)}\right]^{t}=-\frac{1}{r^{2}} \frac{\partial}{\partial r}\left\{r^{2}\left[\delta F_{1-\Delta}^{(1)}\right]_{t r}\right\} & -\frac{1}{r^{2} \sin \theta} \frac{\partial}{\partial \theta}\left\{\sin \theta\left[\delta F_{1-\Delta}^{(1)}\right]_{t \theta}\right\} \\
& +\frac{1}{r^{2} \sin \theta} \frac{\partial}{\partial \theta}\left\{\sin \theta\left(g^{t t}+1\right)\left[\delta F_{0-\Delta}^{(1)}\right]_{t \theta}\right\} .
\end{aligned}
$$

Here $g^{t t}+1=-2 G M / r$ to lowest order.

Our principal goal here is to work out the second-order current $\left[\delta J_{1-\Delta}^{(2)}\right]^{\phi}$. This may be obtained from

$$
\left[\delta J_{0-\Delta}^{(1)}\right]^{\mu}\left[\delta F_{1-\Delta}^{(1)}\right]_{\mu \nu}+\left[\delta J_{1-\Delta}^{(1)}\right]^{\mu}\left[\delta F_{0-\Delta}^{(1)}\right]_{\mu \nu}=-\left[\delta J_{1-\Delta}^{(2)}\right]^{\phi}\left[F_{0-\Delta}^{(0)}\right]_{\phi \nu},
$$

by substituting Eqs. (110), (111), (116), (118), (122) and (124). The resulting expression is fairly complicated, but it simplifies dramatically when the gravitating mass is concentrated at a radius much smaller than the transverse scale $R_{0}$ of the incident Alfvén wave. In this regime, one has $\delta r / r \sim G(d M / d r) \ll G M / r$, and the dominant field and current perturbations are

$$
\begin{aligned}
{\left[\delta F_{1-\Delta}^{(1)}\right]_{\theta r} } & \simeq \frac{2 G M}{r}\left[\delta F_{0-\Delta}^{(1)}\right]_{\theta r} \\
& =\left(\frac{2 G M}{r}\right) \frac{4 \pi R_{0}^{2}}{\sin \theta}\left[1-e^{-R^{2} / 2 R_{0}^{2}}\right] \delta J_{0}(r \cos \theta-t)
\end{aligned}
$$

and

$$
\left[\delta J_{1-\Delta}^{(1)}\right]^{t} \simeq \frac{2 G M}{r}\left[\cos ^{2} \theta e^{-R^{2} / 2 R_{0}^{2}}-\frac{R_{0}^{2}}{r^{2}}\left(1-e^{-R^{2} / 2 R_{0}^{2}}\right)\right] \delta J_{0}(r \cos \theta-t) .
$$

Combining the terms on the left-hand side of Eq. (125), and substituting the background field (19), we find

$$
\left[\delta J_{1-\Delta}^{(2)}\right]^{\phi}=\frac{8 \pi\left(\delta J_{0}\right)^{2}}{B_{0} \sin ^{2} \theta}\left(\frac{G M}{r}\right)\left(\frac{R_{0}}{r}\right)^{4}\left(1-e^{-R^{2} / 2 R_{0}^{2}}\right)^{2}\left[1+\frac{R^{2} / R_{0}^{2}}{e^{R^{2} / 2 R_{0}^{2}}-1}\right] .
$$

This expression simplifies further at small cylindrical radius $\left(R \ll R_{0}\right)$,

$$
\left[\delta J_{1-\Delta}^{(2)}\right]^{\phi} \simeq 6 \pi \frac{\left|\delta J_{0}\right|^{2}}{B_{0}}\left(\frac{G M}{r}\right) \sin ^{2} \theta e^{2 i \omega(z-t)},
$$

when the incident Alfvén wave is a pure harmonic. The second-order current is the source for an outgoing compressive wave

$$
\frac{\partial^{2}\left(r \delta r_{I}\right)}{\partial t^{2}}-\frac{1}{r^{2} \sin \theta} \frac{\partial}{\partial \theta}\left(\sin \theta \frac{\partial\left(r \delta r_{I}\right)}{\partial \theta}\right)-\frac{\partial^{2}\left(r \delta r_{I}\right)}{\partial r^{2}}=\frac{4 \pi r^{2}}{B_{0}}\left[\delta J_{1-\Delta}^{(2)}\right]^{\phi}
$$

of amplitude $\delta r_{I}$. As in the case of a torsional wave propagating in a cylindrical spacetime, the compressive mode is second harmonic and second-order in the wave amplitude.

The Greens function solution to Eq. (130) is dominated by the fluid at radius $r \sim R_{0}$. As a result, the shape of the asymptotic fast wave is sensitive to the distribution of current within the incoming Alfvén mode. We will not examine the pattern of the outgoing compressive wave in full detail here.

\section{COLLISIONS OF AXISYMMETRIC WAVES IN A CYLINDRICAL SPACETIME}

In this section, we study the collision of two torsional Alfvén waves in a uniform magnetofluid in a static cylindrical spacetime $\left(g_{t \phi}=0\right)$. The effects of spacetime curvature are subtle enough that we specialize to axisymmetric modes, supported only by a perturbation $\delta \phi$. The result easily generalizes to the case of flat space, and in the next section we examine more general types of wave interactions in a Minkowski background.

The two Alfvén waves, propagating oppositely along the magnetic field, are labeled + and - , and the component of the current generated by their interaction is labelled $I$. The colliding waves are each assumed to be supported within a cylindrical shell, between radii $R_{\min }$ and $R_{\max }$. We start from the force-free equation

$$
\left(J_{+}^{t}+J_{-}^{t}\right)\left(\delta F_{t r}^{+}+\delta F_{t r}^{-}\right)+\left(J_{+}^{z}+J_{-}^{z}\right)\left(\delta F_{z r}^{+}+\delta F_{z r}^{-}\right)+J_{I}^{\phi} F_{\phi r}^{(0)}=0 .
$$


The background magnetic field is defined by $J^{\phi(0)}=0$ [Eq. (32)]. The fluctuating fields are

$$
\begin{aligned}
\delta F_{z r}{ }^{ \pm} & =\frac{\partial \phi_{ \pm}}{\partial z} F_{\phi r}^{(0)} \\
\delta F_{t r}{ }^{ \pm} & =\frac{\partial \phi_{ \pm}}{\partial t} F_{\phi r}^{(0)}=\mp v_{z} \delta F_{z r}{ }^{ \pm},
\end{aligned}
$$

where $v_{z}$ is given by Eq. (80). The associated currents are

$$
J_{ \pm}^{t}=\frac{1}{4 \pi \sqrt{-g}} \frac{\partial}{\partial r}\left(\sqrt{-g} g^{t t} g^{r r} \delta F_{t r}^{ \pm}\right)= \pm \frac{1}{4 \pi \sqrt{-g}} \frac{\partial}{\partial r}\left(\sqrt{-g} \frac{g^{z z} g^{r r}}{v_{z}} \delta F_{z r}^{ \pm}\right)
$$

and

$$
J_{ \pm}^{z}=\frac{1}{4 \pi \sqrt{-g}} \frac{\partial}{\partial r}\left(\sqrt{-g} g^{z z} g^{r r} \delta F_{z r}^{ \pm}\right) .
$$

Combining these expressions, the force-free equation becomes

$$
\begin{aligned}
-\frac{4 \pi}{B_{0}} \sqrt{-g} J_{I}^{\phi}= & \frac{g_{r r}}{g_{t t}} \frac{\partial^{2} \delta r}{\partial t^{2}}+\frac{g_{r r}}{g_{z z}} \frac{\partial^{2} \delta r}{\partial z^{2}}+\frac{\partial}{\partial r}\left[\frac{\sqrt{-g}}{g_{\phi \phi} g_{r r}} \frac{\partial}{\partial r}\left(\delta r \frac{g_{r r} g_{\phi \phi}}{\sqrt{-g}}\right)\right] \\
= & -2\left(\frac{g_{z z}}{g_{\phi \phi}}\right) \frac{\partial}{\partial r}\left[\left(\frac{g_{\phi \phi}}{g_{z z}}\right)^{2} \frac{\partial \phi_{+}}{\partial z} \frac{\partial \phi_{-}}{\partial z}\right]+ \\
& \left(\frac{g_{\phi \phi}}{g_{z z}}\right)\left[2 \frac{\partial \phi_{+}}{\partial z} \frac{\partial \phi_{-}}{\partial z}-\left(\frac{\partial \phi_{+}}{\partial z}\right)^{2}-\left(\frac{\partial \phi_{-}}{\partial z}\right)^{2}\right] \partial_{r}\left(\ln v_{z}\right) .
\end{aligned}
$$

In contrast with the case of a single (unidirectional) Alfvén wave, a source term appears even when the wave speed has a vanishing gradient $\left(\partial_{r} v_{z}=0\right)$. For that reason we must, in general, retain the curvature corrections in the wave operator for $\delta r$.

\section{A. Collision in a nearly flat, cylindrically symmetric spacetime}

We now specialize to a nearly flat cylindrical spacetime with Newtonian potential $\lambda$ and metric (34), and focus on the source terms in Eq. (135) that are proportional to $\left(\partial_{z} \phi_{+}\right)\left(\partial_{z} \phi_{-}\right)$. The torsional wave speed is $v_{z}=1+2 \lambda$. Comparing with flat space, the new effects we are interested in are already present in the case of a very long wavelength twist in the magnetic field,

$$
\left|\frac{\partial \phi_{ \pm}}{\partial z}\right| R_{\max } \ll 1
$$

so we neglect the derivatives in $t$ and $z$. The perturbation to $B^{z}$ is

$$
\frac{\delta B^{z}}{B_{0}}=\frac{\delta F_{\phi r}^{I}}{F_{\phi r}^{(0)}}=\frac{1}{r} \frac{\partial(r \delta r)}{\partial r}-2 \frac{d \lambda}{d r} \delta r
$$

and the equation of motion (135) simplifies to

$$
\frac{\partial}{\partial r}\left(\frac{\delta B^{z}}{B_{0}}\right)=-\frac{2}{r^{2}} \frac{\partial}{\partial r}\left(r^{4} \frac{\partial \phi_{+}}{\partial z} \frac{\partial \phi_{-}}{\partial z}\right)+4 r^{2} \frac{d \lambda}{d r} \frac{\partial \phi_{+}}{\partial z} \frac{\partial \phi_{-}}{\partial z}
$$

Our first goal is to calculate the amplitude $\delta R_{\max }(t) \equiv \delta r\left(R_{\max }, t\right)$ of the hydrodynamic fluctuation at the outer boundary of the annulus supporting the Alfvén waves. The amplitude of the fast mode at large radius can then easily be obtained, to leading order in $\lambda$, from the equation of motion (61) in Minkowski space. Taking the two waveforms to be 18

$$
\delta \phi_{ \pm}(t, r, z)=\delta \phi_{0 \pm} \sin [k(z \mp t)] f_{\perp \pm}(r)
$$


one sees that the interaction term

$$
\frac{\partial \phi_{+}}{\partial z} \frac{\partial \phi_{-}}{\partial z}=\frac{1}{2} k^{2} \delta \phi_{0+} \delta \phi_{0-}[\cos (2 k t)+\cos (2 k z)] f_{\perp+}(r) f_{\perp-}(r)
$$

is the sum of a uniform oscillation of the cylinder, and a static deformation that excites no wave motion outside the cylinder. Thus the calculation of the fast mode amplitude reduces to the problem of calculating the amplitude $\delta R_{\max }$ of the oscillation at the outer boundary of the cylinder.

We start by integrating Eq. (137) outward from $\delta R=0$ at $r=0$ to obtain

$$
R_{\min } \delta R_{\min }=\left.\left(\frac{1}{2} R_{\min }^{2}+\int_{0}^{R_{\min }} \frac{d \lambda}{d r} r^{2} d r\right) \frac{\delta B^{z}}{B_{0}}\right|_{R_{\min }},
$$

to first order in $\lambda$. The expansion of the magnetofluid is nearly incompressible in the near zone outside the cylinder $(k r \ll 1)$, so the relevant inner and outer boundary conditions are

$$
\frac{\delta B^{z}}{B_{0}} \sim\left(k R_{\max }\right)^{2} \frac{\delta R_{\max }}{R_{\max }} \simeq 0,
$$

and

$$
\left.\frac{\partial \phi_{ \pm}}{\partial z}\right|_{R_{\max }}=\left.\frac{\partial \phi_{ \pm}}{\partial z}\right|_{R_{\min }}=0
$$

The field perturbation is nearly uniform inside radius $R_{\min }$; we will only need the leading term

$$
\left.\frac{\delta B^{z}}{B_{0}}\right|_{R_{\min }}=0+2 \int_{R_{\min }}^{R_{\max }} \frac{1}{r^{2}} \frac{\partial}{\partial r}\left(r^{4} \frac{\partial \phi_{+}}{\partial z} \frac{\partial \phi_{-}}{\partial z}\right) d r=4 \Phi_{+-}\left(R_{\max }\right)
$$

where

$$
\Phi_{+-}(r) \equiv \int_{R_{\min }}^{r} r \frac{\partial \phi_{+}}{\partial z} \frac{\partial \phi_{-}}{\partial z} d r .
$$

The radial perturbation within the annulus $R_{\min }<r<R_{\max }$ can be found iteratively from

$$
\begin{aligned}
r \delta r & -R_{\min } \delta R_{\min }=\int_{R_{\min }}^{r} r^{\prime}\left[\frac{\delta B^{z}}{B_{0}}+2 \frac{d \lambda}{d r^{\prime}} \delta r\left(r^{\prime}\right)\right] d r^{\prime} \\
& =-\left.\frac{1}{2} R_{\min }^{2} \frac{\delta B^{z}}{B_{0}}\right|_{R_{\min }}+\frac{1}{2} r^{2} \frac{\delta B^{z}}{B_{0}}-\int_{R_{\min }}^{r} \frac{1}{2} r^{\prime 2} \frac{\partial}{\partial r^{\prime}}\left(\frac{\delta B^{z}}{B_{0}}\right) d r^{\prime}+2 \int_{R_{\min }}^{r} r^{\prime} \frac{d \lambda}{d r^{\prime}} \delta r\left(r^{\prime}\right) d r^{\prime},
\end{aligned}
$$

after substituting Eq. (138) in the third term on the right-hand side. Evaluating this expression at $r=R_{\max }$ we find

$$
R_{\max } \delta R_{\max }=-2 \int_{R_{\min }}^{R_{\max }} r^{2} \frac{d \lambda}{d r}\left(2 \Phi_{+-}(r)+r \frac{d \Phi_{+-}}{d r}\right) d r+4 \Phi_{+-}\left(R_{\max }\right) \int_{0}^{R_{\max }} r^{2} \frac{d \lambda}{d r} d r .
$$

The first remark to make about this expression is that $\delta R_{\max }$ vanishes in flat space $(d \lambda / d r=$ constant). The same turns out to be true in the special case of a cylindrical line mass $(d \lambda / d r=K / r)$, but not for a more general cylindrical mass distribution that is extended in radius.

We now obtain the fast mode amplitude in the wave zone $k r \gg 1$. The cylindrical wave equation for $\delta B^{z}$ has the outgoing wave solution

$$
\frac{\delta B^{z}}{B_{0}}=A e^{-i k t} H_{0}{ }^{(1)}(k r) \sim A \sqrt{\frac{2}{\pi k r}} e^{i k(r-t)-i \pi / 4} \quad(k r \gg 1)
$$

in Minkowski space. The Hankel function scales as $H_{0}^{(1)}(k r) \sim(2 i / \pi) \ln (k r)$ at small radius. To obtain the normalization factor, we note that in the near zone $(k r \ll 1)$ where the magnetofluid is nearly incompressible, the rate of transport of magnetic flux is nearly constant in radius,

$$
\frac{\partial \Phi}{\partial t}=2 \pi r \frac{\partial(\delta r)}{\partial t} B^{z} \simeq \text { constant } \quad(k r \ll 1) .
$$


This implies

$$
\frac{\partial \delta r}{\partial t}=\left[\frac{\partial(\delta r)}{\partial t}\right]_{R_{\max }}\left(\frac{r}{R_{\max }}\right)^{-1} .
$$

Substituting this expression into $\vec{E}=-\partial_{t}(\delta r) B_{0}(\hat{r} \times \hat{z})$, and thence into

$$
-\frac{\partial \vec{E}}{\partial t}=\vec{\nabla} \times \vec{B}
$$

and integrating over radius, gives

$$
\frac{\delta B^{z}}{B_{0}}-\frac{\delta B^{z}\left(R_{\max }\right)}{B_{0}}=\left[\frac{\partial^{2}(\delta r)}{\partial t^{2}}\right]_{R_{\max }} \ln \left(\frac{r}{R_{\max }}\right) R_{\max }
$$

and

$$
A e^{-i k t}=\frac{i \pi}{2}\left(\omega^{2} R_{\max }\right) \delta r\left(R_{\max }, t\right)
$$

The general solution is

$$
\frac{\delta B^{z}(r, t)}{B_{0}}=-R_{\max } \int_{-\infty}^{t-r} \frac{\partial^{2}(\delta r)}{\partial t^{\prime 2}} \frac{d t^{\prime}}{\sqrt{\left(t-t^{\prime}\right)^{2}-r^{2}}}
$$

\section{B. Collision in a static black string spacetime}

As another illustrative example, we consider the collision between two torsional waves in the spacetime of a static black string [Eq. (37) with $J=0$ ]. The background magnetic field $F_{r \phi}^{(0)}=B_{0} r /\left(r^{2} / \ell^{2}-M\right)$ is aligned with the axis of the string. We assume, as before, that the colliding waves are supported only within a cylindrical annulus $R_{\min }<r<R_{\max }$, and take $R_{\min }, R_{\max } \sim \ell$.

A radial disturbance of frequency $\omega \ll \ell^{-1}$ accumulates a phase

$$
\phi \sim \omega \int\left(-\frac{g_{r r}}{g_{t t}}\right)^{1 / 2} d r=\omega \int \frac{d r}{r^{2} / \ell^{2}-M} .
$$

This integral converges at large radius but diverges near the horizon $r_{H}=M^{1 / 2} \ell$. When $M \ll(\omega \ell)^{2}$, there is a well defined wave zone which is localized at a small radius, $r \lesssim \omega \ell^{2}$. The effect of a small finite angular momentum $J$ is to impose a reflecting barrier for the magnetosonic wave at a radius $r \sim\left(J^{2} / 4 \omega\right)^{1 / 3}$, and therefore to form a resonant cavity. We will not consider the case of finite angular momentum here.

The medium outside radius $R_{\max }$ will, at the same time, respond to a low-frequency disturbance with a uniform compression or rarefaction, $\delta F_{\phi r} \sim F_{\phi r}^{(0)}$. Thus $\delta r \sim r$ at a large radius $(r \gg \ell)$. The amplitude of the radial perturbation $\delta R_{\max }$ can be obtained by noting that the magnetic flux

$$
\Phi=\int 2 \pi d r F_{r \phi}=\int d r \frac{2 \pi r B_{0}}{r^{2} / \ell^{2}-M}
$$

diverges at large radius. At the same time, the background field energy

$$
\frac{d E_{B}}{d z}=\int 2 \pi d r \sqrt{g_{r r} g_{\phi \phi}}\left[-g^{t t} g^{r r} g^{\phi \phi} \frac{F_{r \phi}^{2}}{8 \pi}\right]=\int \frac{B_{0}^{2}}{4} \frac{r d r}{\left(r^{2} / \ell^{2}-M\right)^{5 / 2}}
$$

is finite. As a result, an oscillation $\delta R_{\max }$ of the outer boundary of the annulus is accompanied by a small field perturbation, $\delta B_{z}\left(R_{\max }\right) \simeq 0$.

The nature of the wave solutions at small radius is most easily demonstrated by taking the limit $M \rightarrow 0, Z(r) \rightarrow$ $r^{2} / \ell^{2}$. Then the wave equation (75), which applies to the case of a uniform radial oscillation $\delta r\left(R_{\max }, z, t\right) \propto e^{-i \omega t}$, simplifies considerably. It transforms under the change of variable $r_{*}=-\ell^{2} / r$ to

$$
-\frac{\partial^{2}}{\partial t^{2}}\left(\frac{\delta B^{z}}{B^{z}}\right)+r_{*} \frac{\partial}{\partial r_{*}}\left[\frac{1}{r_{*}} \frac{\partial}{\partial r_{*}}\left(\frac{\delta B^{z}}{B^{z}}\right)\right]=0 .
$$


A further change of variable $\delta B^{z} / B^{z}=h\left(r_{*}\right)\left(\omega r_{*}\right) e^{-i \omega t}$ yields the Bessel equation

$$
r_{*}^{2} \frac{\partial^{2} h}{\partial r_{*}^{2}}+r_{*} \frac{\partial h}{\partial r_{*}}+\left(\omega^{2} r_{*}^{2}-1\right) h=0
$$

which has the wave solution

$$
\frac{\delta B^{z}}{B^{z}}=\left(\omega r_{*}\right) H_{1}^{(2)}\left(\omega r_{*}\right) A e^{-i \omega t} \sim A\left(\omega r_{*}\right) \sqrt{\frac{2}{\pi\left(\omega r_{*}\right)}} e^{-i \omega\left(r_{*}+t\right)-3 \pi / 4}
$$

propagating to small $r$ (large negative $r_{*}$ ). It will be noted that the amplitude of the wave diverges in the wave zone, because the radial phase velocity

$$
\frac{\omega}{k}=\sqrt{-\frac{g_{t t}}{g_{r r}}} \sim\left(\frac{\ell}{r_{*}}\right)^{2}
$$

asymptotes to zero.

To relate the coefficient $A$ to the amplitude of the oscillation at radius $R_{\min }$, we note that the rate of transport of magnetic flux

$$
2 \pi F_{r \phi} \frac{\partial(\delta r)}{\partial t}=\frac{2 \pi B_{0} \ell^{2}}{r} \frac{\partial(\delta r)}{\partial t}
$$

is constant in the near zone $\left(\left|\omega r_{*}\right| \ll 1\right)$. Hence $\partial_{r}(\delta r) \simeq \delta r / r$. From Eq. (73) we have,

$$
\frac{\partial^{2} \delta r_{*}}{\partial t^{2}}=\frac{\partial}{\partial r_{*}}\left(\frac{\delta B^{z}}{B^{z}}\right)
$$

Combining this with the low frequency expansion $\left(\omega r_{*}\right) H_{1}^{(2)}\left(\omega r_{*}\right) \sim 2 i / \pi-(i / \pi)\left(\omega r_{*}\right)^{2} \ln \left(\omega r_{*} / 2\right)$ gives

$$
A e^{-i \omega t} \simeq \frac{-i \pi / 2}{\ln \left(\omega R_{\min }^{*} / 2\right)} \frac{\delta R_{\min }^{*}(t)}{R_{\min }^{*}} .
$$

The last step in obtaining the amplitude of the fast mode that emerges from the wave collision, is to express $\delta R_{\min }$ in terms of the amplitudes of the colliding Alfvén modes. The terms involving $\left(\partial_{z} \phi_{+}\right)\left(\partial_{z} \phi_{-}\right)$in Eq. (135) can be combined to give

$$
\frac{\partial}{\partial r}\left(\frac{\delta B^{z}}{B^{z}}\right)=\frac{\partial}{\partial r}\left[r \frac{\partial}{\partial r}\left(\frac{\delta r}{r}\right)\right]=-\frac{2}{r} \frac{\partial}{\partial r}\left(r^{3} \frac{\partial \phi_{+}}{\partial z} \frac{\partial \phi_{-}}{\partial z}\right)
$$

Integrating with respect to radius, this becomes

$$
\begin{aligned}
\frac{\delta R_{\min }}{R_{\min }}-\frac{\delta R_{\max }}{R_{\max }} & =-\int_{R_{\min }}^{R_{\max }} d r\left\{\frac{\partial}{\partial r}\left[\ln \left(\frac{r}{R_{\min }}\right) \frac{\delta B^{z}}{B^{z}}\right]-\ln \left(\frac{r}{R_{\min }}\right) \frac{\partial}{\partial r}\left(\frac{\delta B^{z}}{B^{z}}\right)\right\} \\
& =2 \int_{R_{\min }}^{R_{\max }} d r r\left[1-\ln \left(\frac{r}{R_{\min }}\right)\right] \frac{\partial \phi_{+}}{\partial z} \frac{\partial \phi_{-}}{\partial z}
\end{aligned}
$$

since $\partial_{z} \phi_{ \pm}\left(R_{\min }\right)=\partial_{z} \phi_{ \pm}\left(R_{\max }\right)=0$ and $\delta B_{z}\left(R_{\max }\right)=0$. The field perturbation at the inner boundary is obtained by substituting $R_{\min } \rightarrow R_{\max }$ in the logarithms in the first line of Eq. (166). After subtracting the two equations, one obtains

$$
\left.\frac{\delta B^{z}}{B^{z}}\right|_{R_{\min }}=2 \int_{R_{\min }}^{R_{\max }} r \frac{\partial \phi_{+}}{\partial z} \frac{\partial \phi_{-}}{\partial z} d r
$$

\section{COLLISIONS OF NON-AXISYMMETRIC WAVES IN MINKOWSKI SPACE}

We will now broaden our discussion of mode collisions to non-axisymmetric modes, and consider both fast and Alfvén modes. Three-mode interactions in the force-free limit were previously considered in [3], using the magnetic 
Lagrangian formalism developed there. We return to this subject and clarify the properties of i) the three-mode coupling between two Alfvén waves; ii) the interaction between two fast waves; and iii) the interaction between a fast wave and an Alfvén wave.

Basic constraints on the mode interactions arise from conservation of energy and momentum. Because the relativistic magnetofluid is inherently compressible, there is guaranteed to be a three-mode coupling between two Alfvén waves and a fast wave [3]. The Alfvén waves $(A 1, A 2)$ satisfy the dispersion relation $\omega_{A}= \pm k_{A}^{z}$; and the fast mode $\omega_{F}=\left|\mathbf{k}_{F}\right|$. Conservation of energy

$$
\omega_{A 1}+\omega_{A 2}=\omega_{F}
$$

and longitudinal momentum

$$
\begin{aligned}
k_{A 1}^{z}+k_{A 2}^{z} & =\omega_{A 1}-\omega_{A 2} \\
& =k_{F}^{z}=\omega_{F} \cos \theta_{F}
\end{aligned}
$$

$\left(\cos \theta_{F}=\mathbf{k}_{F} \cdot \mathbf{B}_{0} / k_{F} B_{0}\right)$ can be combined to give

$$
\cos \theta_{F}=\frac{\omega_{A 1}-\omega_{A 2}}{\omega_{A 1}+\omega_{A 2}} .
$$

Thus in a frame in which the two colliding Alfvén modes have equal frequencies, the fast mode is emitted perpendicular to the background field and with a frequency $\omega_{F}=2 \omega_{A}$ - just as we found in Section $\nabla A$

There is also a three-mode interaction between the two colliding Alfvén waves and a third Alfvén wave. From a kinematic viewpoint, this interaction is non-vanishing only if at least one of the colliding modes has a zero-frequency component (14], 15]), as is easily checked by setting $\cos \theta_{F}=1$ in Eqs. (167) and (168). An alternative description is that the magnetic field lines experience a net displacement (or braiding) across at least one of the colliding wavepackets [16], 3]. (A further requirement for a non-vanishing three-mode interaction, as we detail shortly, is that the colliding modes are non-axisymmetric.)

Next consider the interaction between two fast waves. It is here that a significant difference arises between these two viewpoints. If the background magnetic field suffers a net displacement across the fast wavefront, then a zerofrequency component is present in the fourier decomposition of the field. From a kinematic viewpoint, this zerofrequency component would facilitate a three-wave interaction between the two colliding fast modes and a third fast mode. Two colliding fast waves of finite frequency can generate a third fast wave only if the waves are colinear: only in that case is it possible to satisfy conservation of energy

$$
\omega_{F 1}+\omega_{F 2}=\omega_{F 3}
$$

and momentum

$$
\mathbf{k}_{F 1}+\mathbf{k}_{F 2}=\mathbf{k}_{F 3} .
$$

(Two photons propagating obliquely in vacuum cannot merge to form a single photon, in part because these kinematic conditions cannot be satisfied.) From the same kinematic viewpoint a three-wave interaction between two colliding fast waves and an Alfvén mode is possible only in the degenerate case where both colliding waves propagate along the background magnetic field. [19] This restriction would appear to be somewhat relaxed if one of the waves has a zero-frequency component: then the direction of propagation of that wave can be arbitrary.

As our second task in this section, we calculate the form of this three-mode interaction for two colliding planar fast waves. We find that, in fact, no fast wave is emitted during the collision, but an Alfvén wave is. The Alfvén wave is emitted for any direction of propagation of the two colliding waves. Moreover, when there is a field line displacement in one fast wave, and the other is a pure sinusoid, then the outgoing Alfvén wave is also a pure sinusoid but its frequency is not obtainable from a three-wave resonance condition. It is only when the two colliding modes are directed along the background magnetic field that the resonance condition can be satisfied with a zero-frequency component.

The fast mode also undergoes a four-mode interaction analogous to photon scattering, with the conservation relations

$$
\omega_{F 1}+\omega_{F 2}=\omega_{F 3}+\omega_{F 4}
$$

and

$$
\mathbf{k}_{F 1}+\mathbf{k}_{F 2}=\mathbf{k}_{F 3}+\mathbf{k}_{F 4} .
$$

However, the fast mode also has a non-linear interaction with an Alfvén mode. We show that if the Alfvén wave spectrum is that of a critically balanced cascade (17]), then this second interaction dominates the self-interaction of the fast mode (as was claimed without detailed justification in [3] ). 


\section{A. Collision between two Alfvén waves}

A collision between two axisymmetric torsional modes generates a compressive motion transverse to the axis of the background magnetic field. The amplitude of this compressive motion is, however, strongly suppressed when the colliding wavepackets are highly elongated $\left(k_{z} R_{\max } \ll 1\right)$. In the case of two monochromatic torsional waves with frequencies $\omega=\mp k_{z}$, this radial disturbance vanishes at the outer boundary of the zone supporting the colliding Alfvén waves. More generally, $\delta R_{\max }$ is suppressed by a factor $\sim\left(k_{z} R_{\max }\right)^{2}$, compared with the result of simple dimensional analysis,

$$
\frac{\delta R_{\max }}{R_{\max }} \sim\left(k_{z} R_{\max }\right)^{2} \times R_{\max }^{2} \frac{\partial \phi_{+}}{\partial z} \frac{\partial \phi_{-}}{\partial z}
$$

In other words, the three-mode interaction between two Alfvén waves and the fast mode is suppressed for elongated wavepackets (such as are created during a weak turbulent cascade [17]).

There is, nonetheless, a much stronger three-mode interaction involving a third Alfvén wave. This interaction is not apparent if one restricts the two colliding waves to be axially symmetric, and so we will broaden the analysis here to include Alfvén modes supported by coordinate perturbations $\delta \phi$ and $\delta r$. The relevant component of the force-free equation is

$$
J^{t(1)} F_{t \phi}^{(1)}+J^{z(1)} F_{z \phi}^{(1)}+J^{r(2)} F_{r \phi}^{(0)}=0 .
$$

In Minkowski space, $\delta F_{t \phi}^{(1)}=\left(B_{0} r\right) \partial_{t} \delta r$ and $\delta F_{z \phi}^{(1)}=\left(B_{0} r\right) \partial_{z} \delta r$. The second-order current vanishes for the two separate modes + and - , but where they overlap the first two terms in Eq. (175) do not cancel:

$$
J^{t(1)} F_{t \phi}^{(1)}+J^{z(1)} F_{z \phi}^{(1)}=\frac{B_{0}^{2}}{2 \pi}\left[\partial_{\phi}\left(\partial_{z} \delta r_{+} \partial_{z} \delta r_{-}\right)-\partial_{z} \delta r_{+} \partial_{r}\left(r^{2} \partial_{z} \delta \phi_{-}\right)-\partial_{z} \delta r_{-} \partial_{r}\left(r^{2} \partial_{z} \delta \phi_{+}\right)\right] .
$$

When at least one of the colliding Alfvén wavepackets is not axisymmetric (with both perturbations $\delta \phi$ and $\delta r$ excited), one also finds an explicit second order contribution to the fields,

$$
\delta F_{r t}^{(2)}=\left[\frac{1}{r} \partial_{r}\left(r \delta r_{+}\right) \partial_{t} \delta \phi_{-}+\frac{1}{r} \partial_{r}\left(r \delta r_{-}\right) \partial_{t} \delta \phi_{+}-\partial_{t} \delta r_{+} \partial_{r} \delta \phi_{-}-\partial_{t} \delta r_{-} \partial_{r} \delta \phi_{+}\right] B_{0} r
$$

and similarly for $\delta F_{r \phi}^{(2)}$ and $\delta F_{r z}^{(2)}$. The second-order current therefore has an explicit contribution from the colliding modes. As before, an additional interaction component $\delta r_{I}, \delta \phi_{I}$ to the coordinate fields is required to solve the force-free equation:

$$
\begin{aligned}
4 \pi J^{r(2)} & =-\partial_{t} \delta F_{r t}^{(2)}+\partial_{z} \delta F_{r z}^{(2)}+\frac{1}{r^{2}} \partial_{\phi} \delta F_{r \phi}^{(2)} \\
& =-2 B_{0} r\left[\partial_{z} \delta r_{+} \partial_{r} \partial_{z} \delta \phi_{-}+\partial_{z} \delta r_{-} \partial_{r} \partial_{z} \delta \phi_{+}+\partial_{\phi}\left(\partial_{z} \delta \phi_{+} \partial_{z} \delta \phi_{-}\right)\right]+\frac{1}{r^{2}} \partial_{\phi} \delta F_{r \phi}^{(2)} \\
& +B_{0} r\left\{-\partial_{t}^{2} \delta \phi_{I}+\partial_{z}^{2} \delta \phi_{I}+\partial_{\phi}\left[\frac{1}{r^{3}} \partial_{r}\left(r \delta r_{I}\right)+\frac{1}{r^{2}} \partial_{\phi} \delta \phi_{I}\right]\right\} .
\end{aligned}
$$

Thus Eqs. (175) and (176) combine to give the equation of motion

$$
\begin{aligned}
-\partial_{t}^{2} \delta \phi_{I}+\partial_{z}^{2} \delta \phi_{I} & +\frac{1}{r^{2}} \partial_{\phi}\left[\frac{1}{r} \partial_{r}\left(r \delta r_{I}\right)+\partial_{\phi} \delta \phi_{I}+\frac{1}{B_{0} r} \delta F_{r \phi}^{(2)}\right] \\
& =\frac{2}{r}\left[2 \partial_{z} \delta r_{+} \partial_{r}\left(r \partial_{z} \delta \phi_{-}\right)+2 \partial_{z} \delta r_{-} \partial_{r}\left(r \partial_{z} \delta \phi_{+}\right)+r \partial_{\phi}\left(\partial_{z} \delta \phi_{+} \partial_{z} \delta \phi_{-}\right)-\frac{1}{r} \partial_{\phi}\left(\partial_{z} \delta r_{+} \partial_{z} \delta r_{-}\right)\right] .
\end{aligned}
$$

In this equation, the transverse components of the laplacian on the left-hand side suppress the interaction unless they vanish - that is, unless the the new mode is an Alfvén wave (Eq. 47). Even without making this restriction, the dominant three-mode interaction can be obtained by transforming to the variable

$$
\Gamma_{ \pm} \equiv \oint d \phi \delta \phi_{ \pm}(\phi, r) ; \quad \Gamma_{I} \equiv \oint d \phi \delta \phi_{I}(\phi, r)
$$


Here, the integral is performed at constant cylindrical radius. Applying the operator $\oint d \phi$ to Eq. (179) kills off the last term on each side, and we are left with

$$
-\partial_{t}^{2} \Gamma_{I}+\partial_{z}^{2} \Gamma_{I}=\frac{4}{r} \oint d \phi\left[\partial_{z} \delta r_{+} \partial_{r}\left(r \partial_{z} \delta \phi_{-}\right)+\partial_{z} \delta r_{-} \partial_{r}\left(r \partial_{z} \delta \phi_{+}\right)\right]
$$

Transforming to light-cone variables $z_{ \pm}=z \pm t, \partial_{ \pm}=\frac{1}{2}\left(\partial_{z} \pm \partial_{t}\right)$, the three-mode correction to each Alfvén mode can be calculated from

$$
\begin{aligned}
\left.\partial_{-} \Gamma_{+}\right|_{\text {out }}-\left.\partial_{-} \Gamma_{+}\right|_{\text {in }} & =\int d z_{+} \partial_{+} \partial_{-} \Gamma_{I} \\
& =\frac{1}{r} \oint d \phi\left[\partial_{z} \delta r_{+} \partial_{r}\left(r \partial_{z} \Delta \phi_{-}\right)+\partial_{z} \Delta r_{-} \partial_{r}\left(r \partial_{z} \delta \phi_{+}\right)\right] .
\end{aligned}
$$

The correction to $\Gamma_{-}$is obtained by interchanging + and - in this equation.

As advertised, the resonant three-mode interaction depends on the presence of a net twist (radial displacement) of the magnetofluid across the wavepacket,

$$
\Delta \phi_{ \pm}=\int d z_{\mp} \partial_{z} \delta \phi_{ \pm}\left(z_{\mp}\right) ; \quad \Delta r_{ \pm}=\int d z_{\mp} \partial_{z} \delta r_{ \pm}\left(z_{\mp}\right) .
$$

Because the colliding modes are Alfvén modes, $\Delta r_{ \pm}$is related to $\Delta \phi_{ \pm}$through the condition of incompressibility,

$$
\frac{1}{r} \partial_{r}\left(r \Delta r_{ \pm}\right)+\partial_{\phi} \Delta \phi_{ \pm}=0
$$

The three-mode interaction also depends essentially on the non-axisymmetry of the colliding wavepackets (i.e., on the simultaneous presence of coordinate perturbations $\delta r$ and $\delta \phi$ ). Finally we note that applying the operator $\int d \phi$ to the constraint equation (48) for the fast mode gives

$$
\frac{\partial}{\partial r}\left(r^{2} \int d \phi \delta \phi\right)=0
$$

hence $r^{2} \Gamma=$ constant. As long as $\Gamma$ does not have a singularity at $r=0$, we see that $\Gamma=0$ for the fast mode.

\section{B. Collision between two fast waves}

In order to tackle the collision between two fast waves, we consider the special case of two planar waves. (There are inherent complications associated with the choice of cylindrical geometry, due to the the non-locality of waveforms propagating in two spatial dimensions - in contrast with one or three dimensions, the Greens function is not a delta function.)

Choosing a background magnetic field $\mathbf{B}_{0}=B_{0} \hat{z}$, the perturbed Faraday tensor is

$$
F_{\mu \nu}=\left(\partial_{\mu} x^{0} \partial_{\nu} y^{0}-\partial_{\mu} y^{0} \partial_{\nu} x^{0}\right) B_{0}
$$

Each wave, by itself, is equivalent to a plane electromagnetic wave superposed on a uniform background magnetic field, and the associated coordinate perturbation satisfies the usual wave equation,

$$
x^{0}=x+\delta x_{1,2}^{0}\left(x^{\mu}\right) ; \quad \partial_{\nu} \partial^{\nu}\left[\delta x_{1,2}^{0}\left(x^{\mu}\right)\right]=0 .
$$

We can boost along the background magnetic field into a frame in which one of the colliding fast waves propagates in a direction perpendicular to $\mathbf{B}_{0}$, say

$$
\delta \mathbf{x}_{1}^{0}=\delta x_{1}^{0}(x-t) \hat{x}
$$

A specific example of such a waveform is the harmonic perturbation $\mathbf{k}_{1}=k_{1} \hat{x}, \delta x_{1}^{0}=\delta X_{1} e^{i k_{1}(x-t)}$. Because neither fast mode involves a propagating current in the force-free limit, the interaction vanishes if the electric vectors of the two modes are parallel and their direct superposition involves no violation of the constraint $\delta \mathbf{E} \cdot \mathbf{B}_{0}=0$. Thus the waveform of the second mode is chosen to have a non-vanishing derivative in the $y$-direction. (In general all three components of $\mathbf{k}_{2}$ are non-vanishing.) The corresponding coordinate fluctuation is

$$
\delta \mathbf{x}_{2}^{0}=\delta y_{2}^{0}\left(\mathbf{n}_{2} \cdot \mathbf{x}-t\right)\left[\hat{y}+\frac{n_{2 x}}{n_{2 y}} \hat{x}\right]
$$


where $\mathbf{n}_{2}$ is an arbitrary unit vector. The relative normalization of the fluctuations in $x^{0}$ and $y^{0}$ is fixed by the vanishing of the current, $\partial_{y} \delta x_{2}^{0}-\partial_{x} \delta y_{2}^{0}=0$. We allow for the possibility that there is a non-vanishing displacement across (at least) one of the colliding waveforms, e.g.

$$
\Delta x_{1}^{0}=\int d \ell\left(\delta x_{1}^{0}\right)^{\prime}(\ell) \neq 0 ; \quad \Delta y_{2}^{0}=\int d \ell\left(\delta y_{2}^{0}\right)^{\prime}(\ell) \neq 0 .
$$

The current obtained by superposing $\delta \mathbf{x}^{0}=\delta \mathbf{x}_{1}^{0}+\delta \mathbf{x}_{2}^{0}$ is

$$
4 \pi J_{F F}^{\mu}=\partial_{\nu}\left(\partial^{\mu} x^{0} \partial^{\nu} y^{0}-\partial^{\mu} y^{0} \partial^{\nu} x^{0}\right) B_{0}=\left(\partial_{\nu} \partial^{\mu} \delta x_{1}^{0} \partial^{\nu} \delta y_{2}^{0}-\partial_{\nu} \partial^{\mu} \delta y_{2}^{0} \partial^{\nu} \delta x_{1}^{0}\right) B_{0}
$$

Because the linear currents $J^{\mu(1)}$ vanish, the force-free condition is $J^{x(2)}=J^{y(2)}=0$ to second order in the wave amplitude. We must then introduce an interaction field $x_{I}^{\mu 0}$ and associated current

$$
4 \pi J_{I}^{\mu}=\left[\partial_{y} \partial^{\mu} x_{I}^{0}-\partial_{x} \partial^{\mu} y_{I}^{0}+\delta_{x}^{\mu}\left(\partial_{\nu} \partial^{\nu} y_{I}^{0}\right)-\delta_{y}^{\mu}\left(\partial_{\nu} \partial^{\nu} x_{I}^{0}\right)\right] B_{0}
$$

The conditions

$$
J_{I}^{x}+J_{F F}^{x}=0=J_{I}^{y}+J_{F F}^{y}
$$

correspond to the equations of motion

$$
\begin{aligned}
\partial_{\nu} \partial^{\nu} y_{I}^{0}+ & \partial_{x}\left(\partial_{y} \delta x_{I}^{0}-\partial_{x} \delta y_{I}^{0}\right)= \\
& \left(1-n_{2 x}\right)\left[\left(\delta x_{1}^{0}\right)^{\prime \prime}(x-t) \cdot\left(\delta y_{2}^{0}\right)^{\prime}\left(\mathbf{n}_{2} \cdot \mathbf{x}-t\right)-n_{2 x}\left(\delta x_{1}^{0}\right)^{\prime}(x-t) \cdot\left(\delta y_{2}^{0}\right)^{\prime \prime}\left(\mathbf{n}_{2} \cdot \mathbf{x}-t\right)\right]
\end{aligned}
$$

and

$$
\partial_{\nu} \partial^{\nu} x_{I}^{0}-\partial_{y}\left(\partial_{y} \delta x_{I}^{0}-\partial_{x} \delta y_{I}^{0}\right)=n_{2 y}\left(1-n_{2 x}\right) \cdot\left(\delta x_{1}^{0}\right)^{\prime}(x-t) \cdot\left(\delta y_{2}^{0}\right)^{\prime \prime}\left(\mathbf{n}_{2} \cdot \mathbf{x}-t\right)
$$

It is useful to project these equations onto the outgoing fast $\left(\partial_{x} x_{I}^{0}+\partial_{y} y_{I}^{0}\right)$ and Alfvén $\left(\partial_{x} y_{I}^{0}-\partial_{x} y_{I}^{0}\right)$ modes,

$$
\partial_{\nu} \partial^{\nu}\left(\partial_{x} x_{I}^{0}+\partial_{y} y_{I}^{0}\right)=2\left(1-n_{2 x}\right) n_{2 y}\left(\delta x_{1}^{0}\right)^{\prime \prime}(x-t) \cdot\left(\delta y_{2}^{0}\right)^{\prime \prime}\left(\mathbf{n}_{2} \cdot \mathbf{x}-t\right)
$$

and

$$
\begin{aligned}
& \left(-\partial_{t}^{2}+\partial_{z}^{2}\right)\left(\partial_{x} y_{I}^{0}-\partial_{y} x_{I}^{0}\right)=\left(1-n_{2 x}\right) \quad\left[\left(\delta x_{1}^{0}\right)^{\prime \prime \prime}(x-t) \cdot\left(\delta y_{2}^{0}\right)^{\prime}\left(\mathbf{n}_{2} \cdot \mathbf{x}-t\right)-\right. \\
& \left.\left(n_{2 x}^{2}+n_{2 y}^{2}\right)\left(\delta x_{1}^{0}\right)^{\prime}(x-t) \cdot\left(\delta y_{2}^{0}\right)^{\prime \prime \prime}\left(\mathbf{n}_{2} \cdot \mathbf{x}-t\right)\right] \text {. }
\end{aligned}
$$

To find the outgoing perturbation to the fast wave 1, we start with the Greens function solution to Eq. (196),

$$
\left(\partial_{x} x_{I}^{0}+\partial_{y} y_{I}^{0}\right)(\mathbf{x}, t)=2 n_{2 y}\left(1-n_{2 x}\right) \int d^{3} x^{\prime} \frac{1}{\left|\mathbf{x}-\mathbf{x}^{\prime}\right|}\left(\delta x_{1}^{0}\right)^{\prime \prime}\left(x^{\prime}-t+\left|\mathbf{x}-\mathbf{x}^{\prime}\right|\right) \cdot\left(\delta y_{2}^{0}\right)^{\prime \prime}\left(\mathbf{n}_{2} \cdot \mathbf{x}^{\prime}-t+\left|\mathbf{x}-\mathbf{x}^{\prime}\right|\right)
$$

(using the retarded time $t-\left|\mathbf{x}-\mathbf{x}^{\prime}\right|$ ) and take the limit $x, t \rightarrow \infty$ at $y=z=0$. Each wavepacket is localized within a distance $\sim L$ along its direction of propagation. Transforming $x^{\prime} \rightarrow x^{\prime}-x$ and then setting $\left\{x^{\prime}, y^{\prime}, z^{\prime}\right\}=$ $r\{\sin \theta \cos \phi, \sin \theta \sin \phi, \cos \theta\}$, one has

$$
\begin{array}{r}
\left(\partial_{x} x_{I}^{0}+\partial_{y} y_{I}^{0}\right)(x, 0,0, t)=2 n_{2 y}\left(1-n_{2 x}\right) \int r d r \sin \theta d \theta d \phi\left\{\left(\delta x_{1}^{0}\right)^{\prime \prime}[r(1+\sin \theta \cos \phi)+x-t]\right. \\
\left.\left(\delta y_{2}^{0}\right)^{\prime \prime}\left[r\left(1+\mathbf{n}_{2} \cdot \mathbf{\Omega}\right)+n_{2 x} x-t\right]\right\} .
\end{array}
$$

The integrand is non-vanishing only in a small region $\Delta r \sim L$ near $r=\left(t-n_{2 x} x\right) /\left(1+\mathbf{n}_{2} \cdot \boldsymbol{\Omega}\right)$ and $\Delta \theta \Delta \phi \sim L / t$ near $\theta=\pi / 2, \phi=\pi$. When integrating over $r$, the argument of $\left(\delta x_{1}^{0}\right)^{\prime \prime}$ varies only by a small amount $\sim L^{2} / t$. The integral $\int d \ell\left(\delta y_{2}^{0}\right)^{\prime \prime}(\ell)$ itself vanishes, and so the outgoing fast mode has an amplitude $\sim(L / t)\left(\delta x_{1}^{0}\right)^{\prime}\left(\delta y_{2}^{0}\right)^{\prime} \rightarrow 0$ as $t \rightarrow \infty$. We conclude that the two fast modes do not couple to a third fast mode, even if one of the colliding waveforms has a non-vanishing displacement (190). 
Now let us examine the coupling to an outgoing Alfvén mode. Transforming to the light-cone coordinates $z_{ \pm}=z \pm t$, and taking $x=y=0$, Eq. (197) becomes

$$
\begin{aligned}
\partial_{+} \partial_{-}\left(\partial_{x} y_{I}^{0}-\right. & \left.\partial_{y} x_{I}^{0}\right)\left(0,0, z_{+}, z_{-}\right)= \\
& \frac{1}{4}\left(1-n_{2 x}\right)\left\{\left(\delta x_{1}^{0}\right)^{\prime \prime \prime}\left[\frac{1}{2}\left(z_{-}-z_{+}\right)\right] \cdot\left(\delta y_{2}^{0}\right)^{\prime}\left[\frac{1}{2}\left(n_{2 z}+1\right) z_{-}+\frac{1}{2}\left(n_{2 z}-1\right) z_{+}\right]\right. \\
- & \left.\left(n_{2 x}^{2}+n_{2 y}^{2}\right)\left(\delta x_{1}^{0}\right)^{\prime}\left[\frac{1}{2}\left(z_{-}-z_{+}\right)\right] \cdot\left(\delta y_{2}^{0}\right)^{\prime \prime \prime}\left[\frac{1}{2}\left(n_{2 z}+1\right) z_{-}+\frac{1}{2}\left(n_{2 z}-1\right) z_{+}\right]\right\} .
\end{aligned}
$$

For example, the outgoing Alfvén wave propagating to large positive $z$ is

$$
\begin{aligned}
\partial_{-}\left(\partial_{x} y_{I}^{0}-\right. & \left.\partial_{y} x_{I}^{0}\right)(0,0, z-t)= \\
& \frac{1}{4}\left(1-n_{2 x}\right) \int d z_{+}\left\{\left(\delta x_{1}^{0}\right)^{\prime \prime \prime}\left[\frac{1}{2}\left(z_{-}-z_{+}\right)\right] \cdot\left(\delta y_{2}^{0}\right)^{\prime}\left[\frac{1}{2}\left(n_{2 z}+1\right) z_{-}+\frac{1}{2}\left(n_{2 z}-1\right) z_{+}\right]\right. \\
- & \left.\left(n_{2 x}^{2}+n_{2 y}^{2}\right)\left(\delta x_{1}^{0}\right)^{\prime}\left[\frac{1}{2}\left(z_{-}-z_{+}\right)\right] \cdot\left(\delta y_{2}^{0}\right)^{\prime \prime \prime}\left[\frac{1}{2}\left(n_{2 z}+1\right) z_{-}+\frac{1}{2}\left(n_{2 z}-1\right) z_{+}\right]\right\} .
\end{aligned}
$$

This integral has a closed form solution when one of the wavepackets (say 2) is much shorter than the other and has a zero-frequency component. Then we can treat $\left(\delta y_{2}^{0}\right)^{\prime}$ as a delta function, $\left(\delta y_{2}^{0}\right)^{\prime}(\ell)=\Delta y_{2}^{0} \delta(\ell)$. After restoring the dependence on the transverse coordinates $x$ and $y$, and integrating once over $z_{-}$, our final expression for the outgoing wave is

$$
\left(\partial_{x} y_{I}^{0}-\partial_{y} x_{I}^{0}\right)(x, y, z-t)=\frac{\left(1-n_{2 x}\right)}{2 n_{2 z}} \Delta y_{2}^{0} \cdot\left(\delta x_{1}^{0}\right)^{\prime \prime}\left[\frac{1-n_{2 x}-n_{2 z}}{1-n_{2 z}} x-\frac{n_{2 y}}{1-n_{2 z}} y-\frac{n_{2 z}}{1-n_{2 z}}(z-t)\right] .
$$

The outgoing Alfvén wave is sheared, with wavevector proportional to

$$
(\omega, \mathbf{k}) \propto\left(n_{2 z}, n_{2 x}+n_{2 z}-1, n_{2 y}, n_{2 z}\right) .
$$

Notice also that the amplitude of the outgoing wave involves a higher-order derivative of the ingoing wave, and so the higher-wavenumber fourier components are enhanced by the collision.

The frequency of the outgoing Alfvén wave is

$$
\omega_{A}=\frac{n_{2 z}-n_{1 z}}{n_{2 z}-1} \omega_{F 1}
$$

when the fast wave 1 is a pure fourier mode with frequency $\omega_{F 1}$, and its wavevector has some component along the background magnetic field. The three-wave resonance condition

$$
\omega_{A}=\omega_{F 1}+\omega_{F 2}=\omega_{F 1} ; \quad k_{A}^{z}=k_{F 1}^{z}+k_{F 2}^{z}=k_{F 1}^{z},
$$

can be satisfied with $\omega_{F 2}=k_{F 2}^{z}=0$ if the propagation of fast wave 1 is aligned with the background field $\left(n_{1 z}=1\right)$. But, more generally, Eq. (204) is not consistent with such a three-wave resonance: the integral over $z_{+}$in Eq. (201) is non-vanishing only if

$$
\left(n_{1 z}-1\right) \omega_{F 1}+\left(n_{2 z}-1\right) \omega_{F 2}=0 .
$$

A solution to this equation, with at least one non-vanishing frequency, requires that either $n_{1 z}=1$ or $n_{2 z}=1$. This illustrates why a net field line displacement across an MHD wave has only a limited description in terms of a zero-frequency component of the wave.

\section{Collision between a fast wave and an Alfvén wave}

The collision between a fast wave and an Alfvén wave can generate both an outgoing Alfvén mode and an outgoing fast mode through three-wave interactions. It is useful to compare the strength of this interaction with the interaction 
between two fast modes discussed in the previous section. We focus on the particular case where the Alfvén wave spectrum is highly anisotropic, and the coupling parameter $k_{y} x^{0}-k_{x} y^{0} \sim\left(k_{\perp} / \omega\right)\left(\delta B_{A, \omega} / B\right)$ is independent of scale and close to unity. A key difference between these two types of wave collisions is that the sheared Alfvén waves are current-carrying, $J_{A, \omega}^{(1)} \sim k_{\perp} \delta B_{A, \omega}$ and there is a second-order Lorentz force involving the interaction between this current and the fluctuating field of the fast wave. The magnitude of this Lorentz force is

$$
4 \pi J_{A, \omega}^{(1)} \delta B_{F, \omega} \sim k_{\perp} \delta B_{A, \omega} \delta B_{F, \omega} .
$$

Here $\delta B_{A, \omega}$ and $\delta B_{F, \omega}$ are the field perturbations of the Alfvén and fast waves at frequency $\omega$. We can compare this with the second order current generated in the fast-wave collision,

$$
4 \pi J_{F F}^{(2)} \sim \omega \frac{\delta B_{F, \omega}^{2}}{B_{0}} .
$$

The ratio is

$$
\frac{J_{A, \omega}^{(1)} \delta B_{F, \omega}}{J_{F F}^{(2)} B_{0}} \sim\left(\frac{k_{\perp} \delta B_{A, \omega}}{\omega B_{0}}\right)\left(\frac{\delta B_{F, \omega}}{B_{0}}\right)^{-1} \sim\left(\frac{\delta B_{F, \omega}}{B_{0}}\right)^{-1} \gg 1 .
$$

Thus, if the Alfvén waves form an anisotropic cascade, then the fast wave spectrum evolves predominantly through collisions with Alfvén waves and not through self-collisions between fast waves.

\section{Acknowledgments}

We thank the NSERC of Canada for its financial support, and Maxim Lyutikov for conversations.

\section{APPENDIX A}

In this appendix, we show how the force-free equation may be derived from the action

$$
S^{\prime}=\int d^{4} x_{0} L^{\prime}=\int d^{4} x \frac{1}{4} \sqrt{-g} g_{\mu \rho} g_{\nu \sigma} \widetilde{F}^{\mu \nu} \widetilde{F}^{\rho \sigma}=\int d^{4} x_{0} \frac{J_{4}}{4} \sqrt{-g} g_{\mu \rho} g_{\nu \sigma} \widetilde{F}^{\mu \nu} \widetilde{F}^{\rho \sigma},
$$

using the magnetic lagrangian fluid variables (10). Varying Eq. (A1),

$$
\delta S^{\prime}=\int d^{4} x_{0} \delta L^{\prime}=\int d^{4} x_{0}\left[\frac{\partial L^{\prime}}{\partial x^{\mu}} \delta x^{\mu}+\frac{\partial L^{\prime}}{\partial\left(\partial x^{\mu} / x_{0}^{\alpha}\right)} \delta\left(\frac{\partial x^{\mu}}{\partial x_{o}^{\alpha}}\right)\right],
$$

there are terms which arise from the explicit $x^{\mu}$-dependence of the metric,

$$
\frac{\partial L^{\prime}}{\partial x^{\mu}}=\left[\frac{\left(-g_{0}\right)}{4 J_{4}} \frac{\partial x^{\beta}}{\partial x_{0}^{\kappa}} \frac{\partial x^{\gamma}}{\partial x_{0}^{\lambda}} \frac{\partial x^{\delta}}{\partial x_{0}^{\eta}} \frac{\partial x^{\varepsilon}}{\partial x_{0}^{\zeta}} \widetilde{F}_{0}^{\kappa \lambda} \widetilde{F}_{0}^{\eta \zeta}\right] \frac{\partial}{\partial x^{\mu}}\left[\frac{g_{\beta \delta} g_{\gamma \varepsilon}}{\sqrt{-g}}\right]
$$

as well as from the dependence of $\widetilde{F}^{\mu \nu}$ on $\partial x^{\mu} / \partial x_{0}^{\alpha}$,

$$
\frac{\partial L^{\prime}}{\partial\left(\partial x^{\mu} / x_{0}^{\alpha}\right)}=\left[\frac{g_{\beta \delta} g_{\gamma \varepsilon}}{\sqrt{-g}}\right] \frac{\partial}{\partial\left(\partial x^{\mu} / x_{0}^{\alpha}\right)}\left[\frac{\left(-g_{0}\right)}{4 J_{4}} \frac{\partial x^{\beta}}{\partial x_{0}^{\kappa}} \frac{\partial x^{\gamma}}{\partial x_{0}^{\lambda}} \frac{\partial x^{\delta}}{\partial x_{0}^{\eta}} \frac{\partial x^{\varepsilon}}{\partial x_{0}^{\zeta}} \widetilde{F}_{0}^{\kappa \lambda} \widetilde{F}_{0}^{\eta \zeta}\right] \text {. }
$$

After extremizing, the usual Euler-Lagrange equations are obtained,

$$
\frac{\partial}{\partial x_{0}^{\alpha}}\left[\frac{\partial L^{\prime}}{\partial\left(\partial x^{\mu} / \partial x_{0}^{\alpha}\right)}\right]-\frac{\partial L^{\prime}}{\partial x^{\mu}}=0
$$

Substituting eqs. A3 and (A4) then gives

$$
\begin{aligned}
& \frac{\partial}{\partial x_{0}^{\alpha}}\left[g_{\beta \delta} g_{\gamma \mu} \widetilde{F}^{\beta \gamma}\left(\sqrt{-g_{0}} \frac{\partial x^{\delta}}{\partial x_{0}^{\eta}} \widetilde{F}_{0}^{\eta \alpha}\right)-\frac{\partial J_{4}}{\partial\left(\partial x^{\mu} / \partial x_{0}^{\alpha}\right)} \frac{\sqrt{-g}}{4} g_{\beta \delta} g_{\gamma \varepsilon} \widetilde{F}^{\beta \gamma} \widetilde{F}^{\delta \varepsilon}\right] \\
& -\frac{(-g) J_{4}}{4} \widetilde{F}^{\beta \gamma} \widetilde{F}^{\delta \varepsilon} \frac{\partial}{\partial x^{\mu}}\left[\frac{g_{\beta \delta} g_{\gamma \varepsilon}}{\sqrt{-g}}\right]=0 .
\end{aligned}
$$


Now the background field satisfies

$$
\frac{\partial}{\partial x_{0}^{\alpha}}\left(\sqrt{-g_{0}} \frac{\partial x^{\delta}}{\partial x_{0}^{\eta}} \widetilde{F}_{0}^{\eta \alpha}\right)=0 .
$$

Making use of this relation, and the identities

$$
\frac{\partial}{\partial x_{0}^{\alpha}}\left[\frac{\partial J_{4}}{\partial\left(\partial x^{\mu} / \partial x_{0}^{\alpha}\right)}\right]=0 ; \quad J_{4} \frac{\partial}{\partial x^{\mu}}=\frac{\partial J_{4}}{\partial\left(\partial x^{\mu} / \partial x_{0}^{\alpha}\right)} \frac{\partial}{\partial x_{0}^{\alpha}}
$$

in Eq. (A6) gives

$$
\widetilde{F}^{\delta \varepsilon} \frac{\partial}{\partial x^{\varepsilon}}\left[g_{\beta \delta} g_{\gamma \mu} \widetilde{F}^{\beta \gamma}\right]-\frac{1}{2} \widetilde{F}^{\delta \varepsilon} \frac{\partial}{\partial x^{\mu}}\left[g_{\beta \delta} g_{\gamma \varepsilon} \widetilde{F}^{\beta \gamma}\right]=0 .
$$

To demonstrate the equivalence of this expression and the usual force free equation, one need only substitute $F_{\alpha \beta}=-\frac{1}{2} \sqrt{-g} \varepsilon_{\alpha \beta \rho \sigma} \widetilde{F}^{\rho \sigma}$ in

$$
J^{\mu} F_{\mu \nu}=\frac{1}{\sqrt{-g}} \frac{\partial}{\partial \rho}\left(\sqrt{-g} g^{\mu \alpha} g^{\rho \beta} F_{\alpha \beta}\right) F_{\mu \nu}=0 .
$$

After making use of the contraction

$$
g^{\mu \alpha} \varepsilon_{\alpha \beta \gamma \delta} \varepsilon_{\mu \nu \rho \sigma}=-4 \frac{g_{\beta \nu} g_{\gamma \rho} g_{\delta \sigma}}{(-g)}\left[\delta_{\beta}^{\varepsilon} \delta_{\gamma}^{\kappa} \delta_{\delta}^{\lambda}-\delta_{\beta}^{\varepsilon} \delta_{\delta}^{\kappa} \delta_{\gamma}^{\lambda}+\delta_{\delta}^{\varepsilon} \delta_{\beta}^{\kappa} \delta_{\gamma}^{\lambda}-\delta_{\delta}^{\varepsilon} \delta_{\gamma}^{\kappa} \delta_{\beta}^{\lambda}+\delta_{\gamma}^{\varepsilon} \delta_{\delta}^{\kappa} \delta_{\beta}^{\lambda}-\delta_{\gamma}^{\varepsilon} \delta_{\beta}^{\kappa} \delta_{\delta}^{\lambda}\right],
$$

Eq. (13) is obtained.

\section{APPENDIX B}

In this appendix we list, for reference, the axisymmetric perturbations of a background magnetofluid satisfying $J^{\mu}=0$, using the electric Lagrangian fluid variables (6).

The field strengths are given by

$$
F_{r \phi}=\left(\frac{\partial r_{0}}{\partial r} \frac{\partial \phi_{0}}{\partial \phi}-\frac{\partial \phi_{0}}{\partial r} \frac{\partial r_{0}}{\partial \phi}\right) F_{r \phi}^{(0)}+\left(\frac{\partial r_{0}}{\partial r} \frac{\partial t_{0}}{\partial \phi}-\frac{\partial t_{0}}{\partial r} \frac{\partial r_{0}}{\partial \phi}\right) F_{r t}^{(0)}
$$

where the background fields $F_{r \phi}^{(0)}$ and $F_{r t}^{(0)}$ are

$$
\begin{gathered}
F_{r \phi}^{(0)}=\frac{B_{0} g_{0 \phi \phi} g_{0 r r}}{\sqrt{-g_{0}} R_{0}} \\
F_{r t}^{(0)}=-\left(\frac{g_{0}^{t \phi}}{g_{0}^{t t}}\right) F_{r \phi}^{(0)} .
\end{gathered}
$$

In a cylindrically symmetric spacetime with $z_{0}=z, \partial \phi_{0} / \partial \phi=1$, and $\partial r_{0} / \partial \phi=\partial t_{0} / \partial \phi=0$, the perturbations $\delta F_{\mu \nu}=F_{\mu \nu}-F_{\mu \nu}^{(0)}$ have the form

$$
\begin{gathered}
\delta F_{r \phi}=\left(\frac{\partial r_{0}}{\partial r}\right) F_{r \phi}^{(0)}\left(r_{0}\right)-F_{r \phi}^{(0)}(r) \\
\delta F_{r t}=\left(\frac{\partial r_{0}}{\partial r} \frac{\partial \phi_{0}}{\partial t}-\frac{\partial \phi_{0}}{\partial r} \frac{\partial r_{0}}{\partial t}\right) F_{r \phi}^{(0)}\left(r_{0}\right)+\left(\frac{\partial r_{0}}{\partial r} \frac{\partial t_{0}}{\partial t}-\frac{\partial t_{0}}{\partial r} \frac{\partial r_{0}}{\partial t}\right) F_{r t}^{(0)}\left(r_{0}\right)-F_{r t}^{(0)}(r) \\
\delta F_{r z}=\left(\frac{\partial r_{0}}{\partial r} \frac{\partial \phi_{0}}{\partial z}-\frac{\partial \phi_{0}}{\partial r} \frac{\partial r_{0}}{\partial z}\right) F_{r \phi}^{(0)}\left(r_{0}\right)+\left(\frac{\partial r_{0}}{\partial r} \frac{\partial t_{0}}{\partial z}-\frac{\partial t_{0}}{\partial r} \frac{\partial r_{0}}{\partial z}\right) F_{r t}^{(0)}\left(r_{0}\right) ;
\end{gathered}
$$




$$
\begin{aligned}
& \delta F_{t \phi}=\left(\frac{\partial r_{0}}{\partial t}\right) F_{r \phi}^{(0)}\left(r_{0}\right) ; \\
& \delta F_{z \phi}=\left(\frac{\partial r_{0}}{\partial z}\right) F_{r \phi}^{(0)}\left(r_{0}\right) ;
\end{aligned}
$$

and

$$
\delta F_{t z}=\left(\frac{\partial r_{0}}{\partial t} \frac{\partial \phi_{0}}{\partial z}-\frac{\partial \phi_{0}}{\partial t} \frac{\partial r_{0}}{\partial z}\right) F_{r \phi}^{(0)}\left(r_{0}\right)+\left(\frac{\partial r_{0}}{\partial t} \frac{\partial t_{0}}{\partial z}-\frac{\partial t_{0}}{\partial t} \frac{\partial r_{0}}{\partial z}\right) F_{r t}^{(0)}\left(r_{0}\right)
$$

Substituting $F_{r t}^{(0)}\left(r_{0}\right)=-\left(g_{0}^{t \phi} / g_{0}^{t t}\right) F_{r \phi}^{(0)}\left(r_{0}\right)$, these equations reduce to

$$
\begin{gathered}
\delta F_{r \phi}=\frac{\partial r_{0}}{\partial r} F_{r \phi}^{(0)}\left(r_{0}\right)-F_{r \phi}^{(0)}(r) \\
\delta F_{r t}=\left[\left(\frac{\partial r_{0}}{\partial r} \frac{\partial \phi_{0}}{\partial t}-\frac{\partial \phi_{0}}{\partial r} \frac{\partial r_{0}}{\partial t}\right)-\left(\frac{\partial r_{0}}{\partial r} \frac{\partial t_{0}}{\partial t}-\frac{\partial t_{0}}{\partial r} \frac{\partial r_{0}}{\partial t}\right)\left(\frac{g_{0}^{t \phi}}{g_{0}^{t t}}\right)\right] F_{r \phi}^{(0)}\left(r_{0}\right)-\left(\frac{g^{t \phi}}{g^{t t}}\right) F_{r \phi}^{(0)}(r) ; \\
\delta F_{r z}=\left[\left(\frac{\partial r_{0}}{\partial r} \frac{\partial \phi_{0}}{\partial z}-\frac{\partial \phi_{0}}{\partial r} \frac{\partial r_{0}}{\partial z}\right)-\left(\frac{\partial r_{0}}{\partial r} \frac{\partial t_{0}}{\partial z}-\frac{\partial t_{0}}{\partial r} \frac{\partial r_{0}}{\partial z}\right)\left(\frac{g_{0}^{t \phi}}{g_{0}^{t t}}\right)\right] F_{r \phi}^{(0)}\left(r_{0}\right) ; \\
\delta F_{t \phi}=\left(\frac{\partial r_{0}}{\partial t}\right) F_{r \phi}^{(0)}\left(r_{0}\right) ; \\
\delta F_{z \phi}=\left(\frac{\partial r_{0}}{\partial z}\right) F_{r \phi}^{(0)}\left(r_{0}\right) ;
\end{gathered}
$$

and

$$
\delta F_{t z}=\left[\left(\frac{\partial r_{0}}{\partial t} \frac{\partial \phi_{0}}{\partial z}-\frac{\partial \phi_{0}}{\partial t} \frac{\partial r_{0}}{\partial z}\right)-\left(\frac{\partial r_{0}}{\partial t} \frac{\partial t_{0}}{\partial z}-\frac{\partial t_{0}}{\partial t} \frac{\partial r_{0}}{\partial z}\right)\left(\frac{g_{0}^{t \phi}}{g_{0}^{t t}}\right)\right] F_{r \phi}^{(0)}\left(r_{0}\right)
$$

For example,

$$
\delta F_{r \phi}=\left[\left(\frac{\partial r_{0}}{\partial r}\right) \frac{g_{0 r r} g_{0 \phi \phi}}{\sqrt{-g_{0}}}-\frac{g_{r r} g_{\phi \phi}}{\sqrt{-g}}\right] B_{0}
$$

\section{APPENDIX C}

This appendix is devoted to a derivation of the force-free equation (100) in a non-static, axially symmetric spacetime. The force-free equation (94) is

$$
\begin{gathered}
{\left[\partial_{r}\left(\sqrt{-g} g^{t t} g^{r r} \delta F_{t r}+\sqrt{-g} g^{t \phi} g^{r r} \delta F_{\phi r}\right)+\partial_{z}\left(\sqrt{-g} g^{t \phi} g^{z z} \delta F_{\phi z}\right)\right] F_{t r}^{(0)}+} \\
{\left[\partial_{r}\left(\sqrt{-g} g^{\phi \phi} g^{r r} \delta F_{\phi r}+\sqrt{-g} g^{\phi t} g^{r r} \delta F_{t r}\right)+\partial_{z}\left(\sqrt{-g} g^{\phi \phi} g^{z z} \delta F_{\phi z}\right)+\right.} \\
\left.\partial_{t}\left(\sqrt{-g} g^{\phi \phi} g^{t t} \delta F_{\phi t}+\sqrt{-g} g^{\phi t} g^{\phi t} \delta F_{t \phi}\right)\right] F_{\phi r}^{(0)}=0
\end{gathered}
$$

where the terms involving $\delta F_{t z}$ have canceled. The terms involving $\delta F_{t r}$ may be combined to give

$$
-\sqrt{-g} g^{t t} g^{r r} \delta F_{t r} \frac{\partial}{\partial r}\left(\frac{-g^{t \phi}}{g^{t t}}\right) F_{r \phi}^{(0)} \text {. }
$$


The terms involving $\delta F_{\phi r}$ combine to give

$$
\partial_{r}\left[\frac{\sqrt{-g}}{g_{r r} g_{\phi \phi}} \frac{\partial}{\partial r}\left(\delta r F_{r \phi}^{(0)}\right)\right] F_{r \phi}^{(0)}-\sqrt{-g} g^{t \phi} g^{r r} \frac{\partial}{\partial r}\left(\frac{-g^{t \phi}}{g^{t t}}\right) \frac{\partial}{\partial r}\left(\delta r F_{r \phi}^{(0)}\right) F_{r \phi}^{(0)} .
$$

Here $\operatorname{det}(t, \phi)=g_{t t} g_{\phi \phi}-g_{t \phi}^{2}$. Lastly, the terms involving $\delta F_{\phi t}$ and $\delta F_{\phi z}$ yield

$$
\frac{\sqrt{-g}}{\operatorname{det}(t, \phi)}\left(\frac{\partial^{2} \delta r}{\partial t^{2}}+\frac{g^{z z}}{g^{t t}} \frac{\partial^{2} \delta r}{\partial z^{2}}\right)\left(F_{r \phi}^{(0)}\right)^{2}
$$

To first order in the coordinate perturbation, the perturbed Maxwell fields are

$$
\begin{gathered}
\delta F_{r \phi}=\frac{\partial}{\partial r}\left[\delta r F_{r \phi}^{(0)}(r)\right] ; \\
\delta F_{r t}=\frac{\partial \delta \tilde{\phi}}{\partial t} F_{r \phi}^{(0)}(r)-\frac{\partial}{\partial r}\left[\delta r \frac{g^{t \phi}(r)}{g^{t t}(r)} F_{r \phi}^{(0)}(r)\right] ; \\
\delta F_{t \phi}=\frac{\partial \delta r}{\partial t} F_{r \phi}^{(0)}(r) ;
\end{gathered}
$$

and

$$
\delta F_{z \phi}=\frac{\partial \delta r}{\partial z} F_{r \phi}^{(0)}(r)
$$

Substituting these expressions and collecting terms, we arrive at

$$
\begin{aligned}
& \frac{1}{\operatorname{det}(t, \phi)}\left[\frac{\partial^{2} \delta r}{\partial t^{2}}+\frac{g^{z z}}{g^{t t}}\right.\left.\frac{\partial^{2} \delta r}{\partial z^{2}}\right]\left(F_{r \phi}^{(0)}\right)^{2}+\frac{1}{\sqrt{-g}} \frac{\partial}{\partial r}\left[\frac{\sqrt{-g}}{g_{\phi \phi} g_{r r}} \frac{\partial}{\partial r}\left(\delta r F_{r \phi}^{(0)}\right)\right] F_{r \phi}^{(0)} \\
&-g^{t t} g^{r r} \frac{\partial}{\partial r}\left(\frac{-g^{t \phi}}{g^{t t}}\right)\left[\frac{\partial \widetilde{\phi}}{\partial t}+\delta r \frac{\partial}{\partial r}\left(\frac{-g^{t \phi}}{g^{t t}}\right)\right]\left(F_{r \phi}^{(0)}\right)^{2}=0 .
\end{aligned}
$$

If we multiply by $\operatorname{det}(\phi, t) g^{t t}$ we obtain equation (100).

\section{APPENDIX D}

This www.arxiv.org manuscript incorporates several minor corrections that will appear as an erratum to the paper as published in the December 2004 issue of Physical Review D.

1) The second term, RHS, Eq. (100) [and the analogous term in Eq. (C9)] was spurious and has been deleted.

2) An algebraic error was corrected in the RHS of Eqns. (181) and (182): the normalization increased by a factor 2 ; and $r^{-4}\left[r^{4}\right] \rightarrow r^{-1}[r]$ in front of [inside] the integral. The intermediate equation (179) also received a correction.

3) In Section V.B, a final step has been added to the derivation of the fast mode amplitude [Eq. (166b)]. The claim [based on Eq. (157)] that $\delta R_{\max }$ can be set to zero was incorrect: the integral for the background field energy in Eq. (157) in fact converges at large radius. As a result, the LHS of Eq. (166) gains a term $-\delta R_{\max } / R_{\max }$, and the final step Eq. (166b) is required.

In addition there were a handful of (non-propagating) corrections to signs and indices.

[1] R. Blandford and R. Znajek, Mon. Not. R. Astron. Soc. 179, 433 (1977).

[2] T. Uchida, Mon. Not. R. Astron. Soc. 291, 125 (1997).

[3] C. Thompson and O. Blaes, Phys. Rev. 57, 3219 (1998).

[4] M. Lyutikov and R. D. Blandford (2003), astro-ph/0312347.

[5] S. Chandrasekhar, The Mathematical Theory of Black Holes (Oxford University Press, 1992).

[6] C. Thompson and R. C. Duncan, Mon. Not. R. Astron. Soc. 275, 255 (1995). 
[7] A. Achterberg, Phys. Rev. A 28, 2449 (1983).

[8] R. Wald, Phys. Rev. D 10, 1680 (1974).

[9] J. L. Synge, Relativity: The General Theory (North-Holland, Amsterdam, 1960).

[10] M. Banados, C. Teitelboim, and J. Zanelli, Phys. Rev. Lett. 69, 1849 (1992).

[11] S. Carlip, Class. Quant. Grav. 12, 2853 (1995).

[12] V. M. Nakariakov, B. Roberts, and K. Murawski, Solar Phys. 175, 93 (1997).

[13] D. Tsiklauri, V. M. Nakariakov, and T. D. Arber, Astron. Astrophys. 395, 285 (2002).

[14] D. Montgomery and W. Matthaeus, Astrophys. J. 447, 706 (1995).

[15] C. Ng and A. Bhattacharjee, Astrophys. J. 465, 845 (1996).

[16] P. Goldreich and S. Sridhar, Astrophys. J. 485, 680 (1997).

[17] P. Goldreich and S. Sridhar, Astrophys. J. 438, 763 (1995).

[18] Generally the wavenumbers $k_{+}$and $k_{-}$are not equal; but the translational invariance of the background magnetofluid in the $z$-direction allows them to be made equal through an appropriate Lorentz boost.

[19] We thank Maxim Lyutikov for an illuminating discussion of this point. 